\title{
Maternal underweight and obesity are associated with placental pathologies in human pregnancy.
}

\author{
${ }^{a}$ Department of Health Sciences, Carleton University \\ ${ }^{\mathrm{b}}$ Department of Pathology and Laboratory Medicine, University of British Columbia \\ ${ }^{\mathrm{c}}$ Health Research Methods, Evidence, and Impact, McMaster University \\ ${ }^{1}$ Correspondence: \\ Dr. Kristin Connor \\ Department of Health Sciences, Carleton University, Ottawa, Ontario, Canada, K1S5B6 \\ E: kristin.connor@carleton.ca, Tel.: +1 613-520-2600 ext. 4202
}

Hailey Scotta ${ }^{\mathrm{a}}$, David Grynspan ${ }^{\mathrm{b}}$, Laura N Anderson ${ }^{\mathrm{c}}$, Kristin L Connor ${ }^{\mathrm{a}, 1}$

\begin{abstract}
Introduction: Maternal underweight and obesity are prevalent conditions, associated with chronic, low-grade inflammation, poor fetal development, and long-term adverse outcomes for the child. The placenta senses and adapts to the pregnancy environment in an effort to support optimal fetal development. However, the mechanisms driving these adaptations, and the resulting placental phenotypes, are poorly understood. We hypothesised that maternal underweight and obesity would be associated with increased prevalence of placental pathologies in term and preterm pregnancies.
\end{abstract}

Methods: Data from 12,154 pregnancies were obtained from the Collaborative Perinatal Project, a prospective cohort study conducted from 1959 to 1974 . Macro and microscopic placental pathologies were analysed across maternal prepregnancy body mass index (BMI) to assess differences in the presence of pathologies among underweight, overweight, and obese BMI groups compared to normal weight reference BMI at term and preterm. Placental pathologies were also assessed across fetal sex.

Results: Pregnancies complicated by obesity had placentae with increased fetal inflammation at preterm and increased maternal inflammation at term. In term pregnancies, increasing maternal BMI associated with increased maternal vascular malperfusion (MVM), odds of an appropriate mature placenta for gestational age, and placental weight, and decreased placental efficiency. Male placentae, independent of maternal BMI, had increased inflammation, MVM, and placental efficiency than female placentae, particularly at term.

Discussion: Maternal underweight and obesity are not inert conditions for the placenta, and the histomorphological changes driven by suboptimal maternal BMI may serve as indicators of adversities experienced in utero and potential predictors of future health trajectories.

Keywords: Underweight, obesity, placental pathology, inflammation, Collaborative Perinatal Project

\section{Introduction}

Maternal underweight and obesity are global health burdens; maternal underweight remains a persisting problem, and the prevalence of obesity in pregnancy continues to rise $\mathrm{e}^{1-3}$. Both conditions have been associated with higher levels of inflammation in the mother, which favour increased inflammation in the placenta ${ }^{4}$, suboptimal nutrient availability to the fetus ${ }^{4}$, and adverse pregnancy and offspring outcomes ${ }^{5,6}$. Yet, the mechanisms that drive these outcomes remain poorly understood.

The placenta is a critical regulator of the fetal environment and can adapt to mitigate harmful exposures, or maladapt to permit their adverse effects $^{7}$. For example, inflammation can reduce placental area ${ }^{8}$ and impair spiral artery remodelling ${ }^{9}$, which can affect nutrient and gas exchange ${ }^{8-11}$ with potential consequences for the developing offspring ${ }^{12-}$ ${ }^{14}$. Additionally, there is some evidence that maternal underweight and obesity may be unfavourable for placental development. Maternal obesity has been associated with chronic villitis ${ }^{15,16}$, both delayed and accelerated placental villous maturation ${ }^{16}$, and increased atheromas and villous infarcts ${ }^{16,17}$, pathologies which have been linked to placental insufficiency ${ }^{18}$, fetal growth restriction, and neurodevelopmental impairment ${ }^{16}$. The effects of maternal undernutrition or low BMI on placental pathologies are less well documented ${ }^{19}$. Animal models of undernutrition have shown evidence of abnormal placental vasculature and decreased fetal blood space, labyrinth and junctional zone area ${ }^{19}$, which may impair placental transfer ${ }^{20}$. The pro-inflammatory state induced by maternal underweight and obesity may also directly influence placental function, such as altered expression of placental nutrient transporters ${ }^{21-23}$. Therefore, it is important to understand even subtle placental phenotypes in these common conditions, as placental structural and functional adaptations may serve as a record of adversities experienced in utero, and could help reveal the mechanisms through which these adversities affect the developing offspring. However, the placental morphological and histopathologic changes induced by maternal underweight and obesity, in the absence of other comorbidities or adverse perinatal events, are poorly characterized.

We hypothesised that, compared to women of normal weight, maternal underweight and obesity prepregnancy would be associated with increased prevalence of placental pathologies in term and preterm pregnancies, and that there would be differences in pathology prevalence based on placental sex. Using data from the Collaborative Perinatal 
Project (CPP), our primary objective was to determine whether placental pathologies were more prevalent in term and preterm pregnancies complicated by suboptimal maternal prepregnancy BMI. Our findings help to quantify placental pathologies in common pregnancy conditions, and uncover the mechanisms linking poor maternal metabolic health with suboptimal fetal growth and development.

\section{Methods}

\section{Study design and population}

Secondary data analysis was conducted using data from the CPP, a prospective cohort study designed to identify relationships between pregnancy and perinatal risk factors and child outcomes (https://catalog.archives.gov/id/606622). The CPP was conducted from 1959 to 1974 at 12 hospitals across the United States, and collected pregnancy data through the prenatal period and delivery, and child outcomes for approximately 58,000 pregnancies $^{24}$.

The primary exposure of interest was maternal prepregnancy BMI, specifically maternal underweight, overweight, and obesity compared to normal weight as the reference category. BMI was classified according to the World Health Organization and American College of Obstetricians and Gynecologists guidelines, where maternal BMI is categorized as underweight $(<18.5)$, normal weight $(18.5-25)$, overweight $(>25-30)$, or obese (>30). To evaluate the associations between maternal BMI and placental pathologies, the study sample was restricted to pregnancies with placental pathology data available, and maternal height and prepregnancy weight available to calculate maternal prepregnancy BMI. We included only singleton births from first pregnancies (parity and gravidity of zero), where fetal sex was documented as male or female. Gestational age below 24 weeks or above 43 weeks were excluded based on the limit of viability $^{25}$ and morphological changes to the placenta such as those induced by cellular senescence ${ }^{26}$, and increased risk for fetal complications in post-term pregnancies ${ }^{27}$. The $\mathrm{CPP}$ calculated gestational age based on the last menstrual period to the nearest week. These selection criteria resulted in a sample of 12,154 pregnancies (Supplementary Figure 1).

\section{Placental pathologies}

The primary outcomes were macroscopic and microscopic placental pathologies (Figure 1). Macroscopic data included placental weight, largest and smallest diameter, thickness, and placental shape. Infant birthweight to placental weight/largest diameter/smallest diameter/thickness ratios were calculated as potential predictors of placental efficiency ${ }^{28,29}$. The top and bottom $0.5 \%$ of raw infant and placental anthropometry data (birthweight, placental weight, and placental dimensions) were excluded to remove biologically implausible data. The umbilical cord was assessed for cord edema and number of vessels, given the increased incidence of a single artery cord with gestational diabetes mellitus $(\mathrm{GDM})^{30}$, a covariate of interest for pregnancies with obesity. Thrombosed fetal vessels and cut surface infarcts were assessed, which may be associated with impaired placental perfusion $^{31}$. For microscopic variables: decidual vessel fibrinoids and atheroma were included, features of malperfusion ${ }^{32}$. Neutrophilic infiltration of the umbilical vein, umbilical artery, cord substance, chorion and amnion membranes, and chorion and amnion of the (fetal side) placental surface were also obtained, which may be indicative of ascending maternal infection ${ }^{33}$. Cut surface calcification was included as an indicator for placental maturation ${ }^{34}$. Syncytium-nuclear clumping, or syncytial knots, and stromal fibrosis are signs of accelerated villous maturation and were thus included ${ }^{35}$. Prescence of Langhans' layer,
Hofbauer cells, and pathological edema were included as indicators of placental immaturity ${ }^{36,37}$. A variable provided for the apparent maturity of the placenta was also included as a marker for appropriate placental development for gestational age. For multivariable analyses, categorical placental pathology variables were collapsed into binary categories (Supplementary Table 1).

We derived additional summary scores for placental inflammation, MVM, and placental immaturity. A maternal inflammation summary score was derived from the following variables: opacity of membranes and neutrophil infiltration of the amnion and chorion membranes and of the placental surface. Where data were available for all constituent variables, the individual variable scores were summed to create a composite score ranging from zero to fourteen, where a higher score represents increased levels of inflammation. Similarly, a fetal inflammation summary score was derived from neutrophilic infiltration of the umbilical vein, artery, and cord substance to create a composite score from zero to nine. A summary score considering features of MVM was derived from: presence of infarcts and syncytium-nuclear clumping to create a score ranging from zero to two, where a higher score indicates increased MVM. A placental immaturity score was derived from the following variables: Langhans' layer, Hofbauer cells, stromal fibrosis, and syncytium-nuclear clumping, to create a score ranging from zero to seven where a higher score indicates a more immature placental phenotype (Supplementary Table 2).

\section{Maternal demographics}

Our primary exposure of interest, maternal prepregnancy BMI, was defined based on measured height and self-reported prepregnancy weight collected at study enrollment. In addition to prepregnancy BMI, maternal demographic data including age, race, education, marital status, income, socioeconomic index, housing density, smoking history, diabetes mellitus status, and gestational weight gain were obtained. The socioeconomic index is a composite numerical index derived from scores for education (of the head of household/chief earner), occupation (of the head of household/chief earner), and family income, ranging from 0.0 9.5 , where 9.5 represents the highest socioeconomic status ${ }^{38}$. Smoking history was provided as the number of cigarettes smoked per day at the time of the interview, from zero cigarettes (including non-smokers) to 60 cigarettes per day. Additional smoking categories included 61 or more cigarettes per day, regular smoker but less than one cigarette per day, and irregular smoker but less than four cigarettes per month. Smoking history was presented as non-smokers (including women currently smoking zero cigarettes per day), light smokers (less than one pack of 20 cigarettes per day), and heavy smokers (one or more packs of 20 cigarettes per day). Diabetes mellitus was presented as presence or absence, where presence included diabetes mellitus reported before pregnancy, during pregnancy, both before and during, during and postpartum, or before, during, and postpartum. Based on current recommendations from the Institute of Medicine (IOM) guidelines (2009), maternal weight gain was categorized as inadequate, adequate, or excessive for singleton pregnancies based on prepregnancy BMI, where the recommended weight gain ranges are $28-40$ pounds, $25-35$ pounds, $15-25$ pounds or 11-20 pounds $^{39}$ for mothers who are underweight, normal weight, overweight, or have obesity, respectively.

\section{Statistical analyses}

\section{Univariate analyses}

Univariate analysis was conducted to evaluate differences in the prevalence of placental pathologies across maternal prepregnancy BMI groups. Given that the presence of some placental pathologies are 
medRxiv preprint doi: https://doi.org/10.1101/2021.06.01.21258127; this version posted June 4, 2021. The copyright holder for this preprint (which was not certified by peer review) is the author/funder, who has granted medRxiv a license to display the preprint in perpetuity.

All rights reserved. No reuse allowed without permission.

A

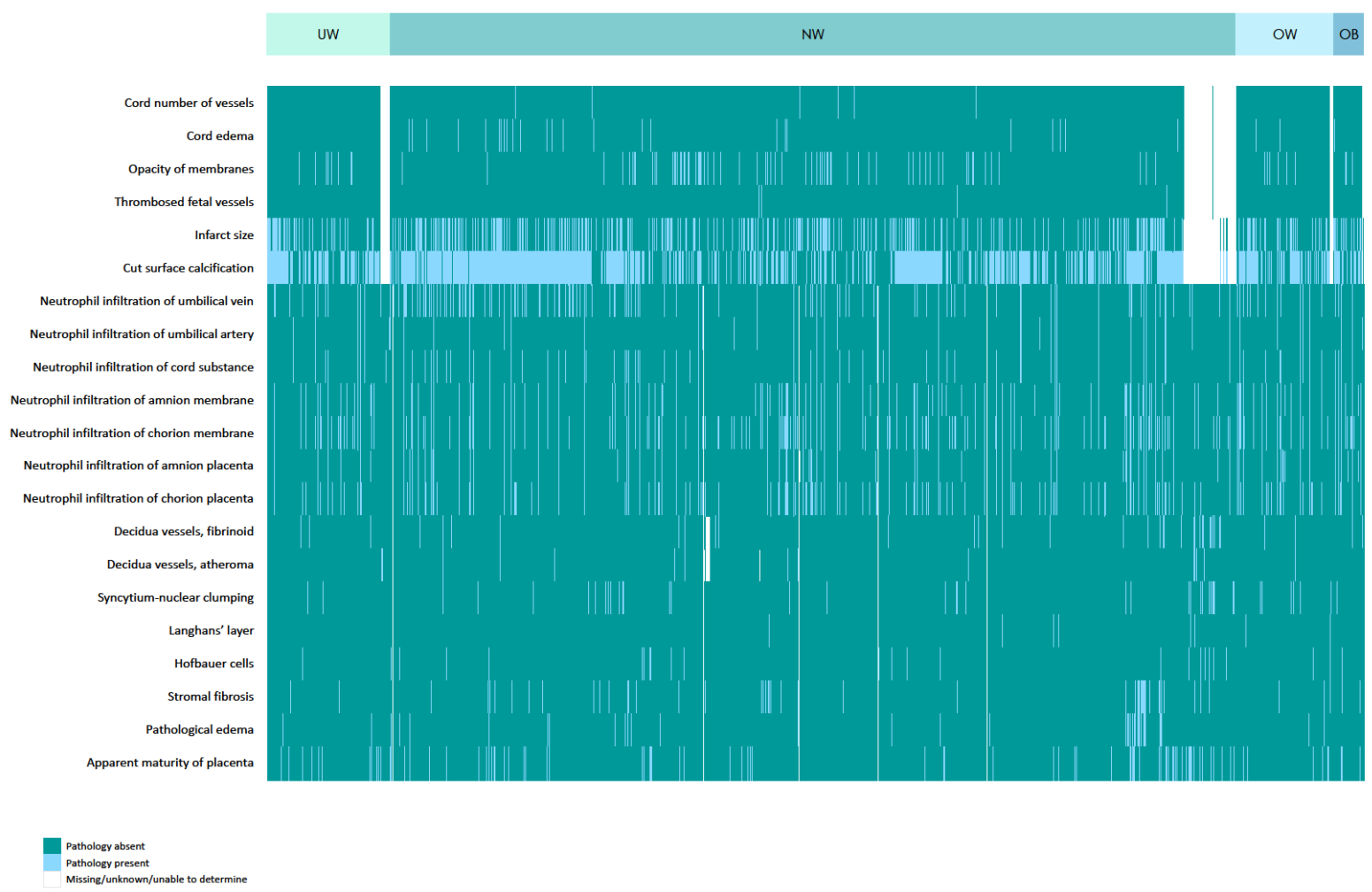

B

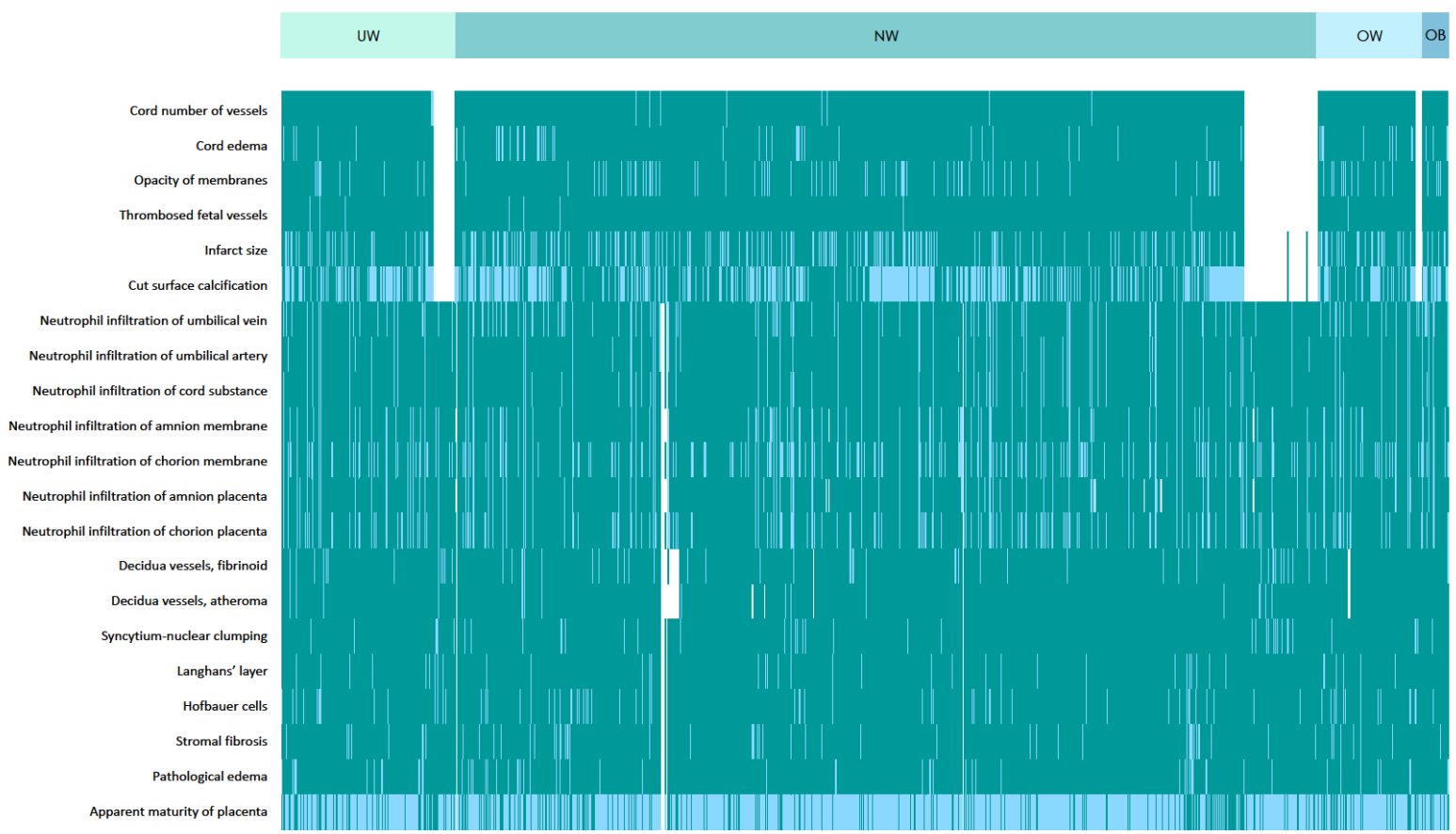

Figure 1. Prevalence of placental pathologies in term (A) and preterm (B) pregnancies. The presence or absence of categorical placental pathologies across maternal body mass index (BMI) groups, where mothers were categorized as underweight (UW), normal weight $(\mathrm{NW})$, overweight $(\mathrm{OW})$ or having obesity $(\mathrm{OB})$ by prepregnancy $B M I$, in term $(n=10,145$; panel $A)$ and preterm $(n=1,739$; panel B) pregnancies. 
dependent on gestational age $e^{40}$, we conducted all analyses stratified for term and preterm placentae. Differences between maternal BMI groups and placental measures were determined by Kruskal-Wallis test with Steel-Dwass post hoc for continuous nonparametric data, and Likelihood Ratio Chi Square test for categorical data. Placental pathologies were also assessed across fetal sex. Data are presented as median (interquartile range) and Wilcoxon test effect size (r) $(95 \%$ confidence interval; $\mathrm{CI}$ ) for non-parametric continuous data, and frequency (percentage) and Cramer's V effect size (95\% CI) or odds ratio $(95 \% \mathrm{CI})$ (for binary fetal sex analyses) for categorical variables. Statistical significance was defined as $\mathrm{p}<0.05$ and all tests were twosided. Data were analysed using JMP statistical software (14.0).

\section{Multivariable analyses}

We performed multivariable logistic and linear regression to determine the relationships between maternal prepregnancy BMI and placental pathologies at term and preterm. Logistic regression models were used to determine the associations between maternal BMI (continuous) and binary placental pathologies. Categorical placental pathology variables were collapsed into binary categories to calculate odds ratios for the pathological phenotype. Any data coded as unknown, unable to determine, or missing were excluded from regression analyses. Data are presented as unadjusted (OR) or adjusted units odds ratio (aOR) (95\% $\mathrm{CI})$. Linear regression models were used to determine the associations between maternal BMI (continuous) and continuous placental variables. Data are reported as adjusted beta coefficient $(\mathrm{a} \beta)(95 \% \mathrm{CI})$. Covariates of interest were identified a priori and included fetal sex (male/female), maternal race (White, Black, and Other), maternal age (continuous), smoking history (non-smoker, light smoker, heavy smoker), maximum gestational weight gain (continuous), diabetes (yes/no), maternal education (continuous), and socioeconomic index (continuous). Two regression models were defined a priori: 1) An unadjusted model was first used to identify the associations between prepregnancy BMI alone (as a continuous variable) with placental pathologies and 2) an adjusted model adjusted for the covariates defined above.

\section{Results}

\section{Maternal demographics differed across maternal BMI groups}

Maximum gestational weight gain among preterm (Wilcoxon test effect size, $\mathrm{r}=-0.09[-0.14$ to -0.04$])$ and term $(\mathrm{r}=-0.05$ [-0.07 to -0.03$])$ pregnancies was greatest in underweight and lowest in obese BMI groups; however, according to IOM (2009) guidelines, weight gain in underweight pregnancies was still inadequate while pregnancies with obesity had excessive weight gain (Supplementary Tables 3-4). Maternal age, race, education, marital status, income, socioeconomic index, and housing density also differed by BMI group (Supplementary Tables 3-4).

\section{Maternal obesity associated with increased neutrophil infiltration of gestational tissues}

Among preterm pregnancies, fetal inflammation, characterised by the composite fetal inflammation summary score, was greater in placentae from mothers with obesity compared to all other BMI groups $(\mathrm{r}=0.009$ [0.04 to 0.05 ], Table 1). There were no effects of maternal BMI group on neutrophil infiltration in the preterm membranes or placental surface. Neutrophil infiltration of the umbilical vein $(\mathrm{aOR}=1.06$ [1.01-1.12]), umbilical cord substance $(\mathrm{aOR}=1.08$ [1.02-1.14]), and amnion membrane $(\mathrm{OR}=1.05$ [1.01-1.10]), but not the umbilical artery, chorion membrane, or amnion and chorion of the placenta, were more likely with increasing maternal BMI (Table 2).
Among term pregnancies, pregnancies complicated by obesity had the greatest percentage of marked neutrophil infiltration of the umbilical vein and artery, and amnion and chorion membranes and of the placental surface, and greatest maternal inflammation summary score compared to underweight ( $\mathrm{r}=0.008$ [-0.01 to 0.03], Table 3). Further, neutrophilic infiltration of the umbilical vein $(\mathrm{aOR}=1.03 \quad[1.01-1.05])$, artery $(\mathrm{aOR}=1.04$ [1.00-1.07]), and cord substance $(\mathrm{aOR}=1.04$ [1.01-1.07]) increased with increasing BMI (Table 4). Neutrophil infiltration of the amnion membrane $(\mathrm{aOR}=1.06$ [1.03-1.08]), chorion membrane $(\mathrm{aOR}=1.04[1.02-1.06])$, amnion of the placental surface $(\mathrm{aOR}=1.04$ [1.01-1.07]), and chorion of the placental surface $(\mathrm{aOR}=1.04$ [1.021.06]) were also greater with increasing BMI (Table 4).

\section{Higher maternal BMI associated with MVM among term pregnancies}

At term, increasing maternal BMI was associated with greater odds of an appropriately mature placenta based on apparent maturity of the placenta $(\mathrm{aOR}=0.94$ [0.92-0.97]) and increased MVM $(\mathrm{a} \beta=0.007$ [0.004-0.01], Table 4), and placentae from mothers with obesity had the greatest percentage of thrombosed fetal vessels compared to all other BMI groups (Cramer's V=0.03 [0.003-0.05], Supplementary Table 5). Additionally, presence of infarcts alone was more likely as maternal BMI increased $(\mathrm{aOR}=1.03$ [1.02-1.05], Supplementary Table 6). There were no differences in apparent placental maturity or vasculature-related pathologies by BMI among preterm pregnancies (Table 1).

\section{Both maternal underweight and increased BMI influenced placental anthropometry}

In preterm pregnancies where mothers were underweight, placental weight was reduced compared to placentae from mothers with overweight and obesity ( $\mathrm{r}=0.04[-0.006-0.09])$, and smallest diameter was reduced compared to placentae from pregnancies complicated by obesity ( $\mathrm{r}=0.06$ [0.009-0.12], Supplementary Table 7), but there were no differences in placental anthropometry compared to normal weight BMI. Among term pregnancies where mothers were underweight, placental weight $(r=0.08$ [0.06-0.10]) and smallest diameter $(r=0.05$ [0.03-0.07]) were reduced compared to all other BMI groups, with most prominent differences between the underweight and obese groups (Supplementary Table 5). At term only, birthweight to placental weight ratio was higher in mothers who were underweight compared to overweight, but not different than normal weight ( $\mathrm{r}=-0.03$ [-0.05 to -0.006$]$, Supplementary Table 5). Similarly, when considering BMI as a continuous variable, placental weight and smallest diameter increased with increasing maternal BMI among preterm and term pregnancies, and birthweight to placental weight ratio decreased with increasing maternal BMI at term (Supplementary Tables 6,8).

\section{Fetal sex influenced placental pathology}

Among preterm pregnancies, female placentae had a greater fetal inflammation summary score than male placentae $(r=-0.05[-0.10$ to 0.004], Table 5), but there were no sex differences in neutrophil infiltration of the membranes or placental surface. Male term placentae had greater fetal and maternal placental inflammation, including neutrophil infiltration of the umbilical vein, umbilical cord substance, amnion membrane, chorion membrane, amnion of the placenta, chorion of the placenta, and fetal and maternal inflammation summary scores (Table 5). Further, when considering only pregnancies with maternal obesity, at term, male placentae had greater neutrophil infiltration of the umbilical vein and chorion membrane than female placentae (Supplementary Figure 2). 
Table 1. Associations between maternal prepregnancy BMI and microscopic placental pathologies in preterm pregnancies, N=1739.

\begin{tabular}{|c|c|c|c|c|c|c|}
\hline \multirow[t]{2}{*}{ Placental pathology } & \multicolumn{4}{|c|}{ Prepregnancy BMI } & \multirow[b]{2}{*}{ p-value } & \multirow[b]{2}{*}{ Effect size } \\
\hline & $\begin{array}{l}\text { UW } \\
(\mathrm{n}=259)\end{array}$ & $\begin{array}{l}\text { NW } \\
(n=1286)\end{array}$ & $\begin{array}{l}\text { OW } \\
(n=156)\end{array}$ & $\begin{array}{l}\text { OB } \\
(n=38)\end{array}$ & & \\
\hline \multicolumn{7}{|l|}{ Cord } \\
\hline Neutrophil infiltration, umbilical vein & & & & & 0.14 & $0.06(0.00-0.07)$ \\
\hline Not seen & $222(85.7)$ & $1126(87.6)$ & $130(83.3)$ & $27(71.1)$ & & \\
\hline Slight & $15(5.79)$ & $71(5.52)$ & $13(8.33)$ & $6(15.8)$ & & \\
\hline Moderate & 0 & $3(0.23)$ & 0 & 0 & & \\
\hline Marked & $7(2.70)$ & $48(3.73)$ & $10(6.41)$ & $3(7.89)$ & & \\
\hline Unknown/missing & $15(5.79)$ & $38(2.95)$ & $3(1.92)$ & $2(5.26)$ & & \\
\hline Neutrophil infiltration, umbilical artery & & & & & 0.62 & $0.04(0.00-0.05)$ \\
\hline Not seen & $228(88.0)$ & $1164(90.5)$ & $143(91.7)$ & $30(78.9)$ & & \\
\hline Slight & $9(3.47)$ & $45(3.50)$ & $3(1.92)$ & $3(7.89)$ & & \\
\hline Moderate & 0 & $1(0.08)$ & 0 & 0 & & \\
\hline Marked & $7(2.70)$ & $37(2.88)$ & $7(4.49)$ & $3(7.89)$ & & \\
\hline Unknown/missing & $15(5.79)$ & $39(3.03)$ & $3(1.92)$ & $2(5.26)$ & & \\
\hline Neutrophil infiltration, cord substance & & & & & 0.23 & $0.05(0.00-0.07)$ \\
\hline Not seen & $233(90.0)$ & $1159(90.1)$ & $141(90.38)$ & $29(76.3)$ & & \\
\hline Slight & $8(3.09)$ & $58(4.51)$ & $6(3.85)$ & $5(13.2)$ & & \\
\hline Moderate & 0 & $2(0.16)$ & 0 & 0 & & \\
\hline Marked & $3(1.16)$ & $28(2.18)$ & $6(3.85)$ & $2(5.26)$ & & \\
\hline Unknown/missing & $15(5.79)$ & $39(3.03)$ & $3(1.92)$ & $2(5.26)$ & & \\
\hline Fetal inflammation summary score & $0(0,0)^{\mathrm{A}}$ & $0(0,0)^{\mathrm{A}}$ & $0(0,0)^{\mathrm{A}}$ & $0(0,1)^{\mathrm{B}}$ & 0.002 & $0.009(-0.04$ to 0.05$)$ \\
\hline \multicolumn{7}{|l|}{ Membranes } \\
\hline Neutrophil infiltration, membrane, amnion & & & & & 0.21 & $0.08(0.03-0.10)$ \\
\hline Not seen & $208(80.3)$ & $1074(83.5)$ & $127(81.4)$ & $29(76.3)$ & & \\
\hline Slight & $21(8.11)$ & $74(5.75)$ & $13(8.33)$ & $5(13.2)$ & & \\
\hline Moderate & 0 & $1(0.08)$ & 0 & $1(2.63)$ & & \\
\hline Marked & $5(1.93)$ & $33(2.57)$ & $6(3.85)$ & $1(2.63)$ & & \\
\hline Unknown/missing & $25(9.65)$ & $104(8.09)$ & $10(6.41)$ & $2(5.26)$ & & \\
\hline Neutrophil infiltration, membrane, chorion & & & & & 0.65 & $0.04(0.00-0.05)$ \\
\hline Not seen & $197(76.1)$ & $1006(78.2)$ & $114(73.1)$ & $27(71.1)$ & & \\
\hline Slight & $27(10.4)$ & $139(10.8)$ & $21(13.5)$ & $3(7.89)$ & & \\
\hline Moderate & $1(0.39)$ & $1(0.08)$ & 0 & 0 & & \\
\hline Marked & $18(6.95)$ & $103(8.01)$ & $16(10.3)$ & $6(15.8)$ & & \\
\hline Unknown/missing & $16(6.18)$ & $37(2.88)$ & $5(3.21)$ & $2(5.26)$ & & \\
\hline Neutrophil infiltration, placental surface, amnion & & & & & 0.62 & $0.04(0.00-0.05)$ \\
\hline Not seen & $208(80.3)$ & $1062(82.6)$ & $127(81.4)$ & $31(81.6)$ & & \\
\hline Slight & $16(6.18)$ & $58(4.51)$ & $8(5.13)$ & $3(7.89)$ & & \\
\hline Moderate & 0 & $1(0.08)$ & 0 & 0 & & \\
\hline Marked & $6(2.32)$ & $25(1.94)$ & $8(5.13)$ & $1(2.63)$ & & \\
\hline Unknown/missing & $29(11.2)$ & $140(10.9)$ & $13(8.33)$ & $3(7.89)$ & & \\
\hline Neutrophil infiltration, placental surface, chorion & & & & & 0.51 & $0.04(0.00-0.06)$ \\
\hline Not seen & $206(79.5)$ & $1061(82.5)$ & $127(81.4)$ & $29(76.3)$ & & \\
\hline Slight & $26(10.0)$ & $118(9.18)$ & $11(7.05)$ & $5(13.2)$ & & \\
\hline Moderate & 0 & 0 & 0 & 0 & & \\
\hline Marked & $12(4.63)$ & $67(5.21)$ & $14(8.97)$ & $2(5.26)$ & & \\
\hline Unknown/missing & $15(5.79)$ & $40(3.11)$ & $4(2.56)$ & $2(5.26)$ & & \\
\hline Maternal inflammation summary score & $0(0,1)$ & $0(0,1)$ & $0(0,1.25)$ & $0(0,2)$ & 0.38 & $0.006(-0.05$ to 0.06$)$ \\
\hline
\end{tabular}




\begin{tabular}{|c|c|c|c|c|c|c|}
\hline Decidua & & & & & & \\
\hline $\begin{array}{l}\text { Decidual vessels, fibrinoid } \\
\text { Not seen } \\
\text { Present } \\
\text { Unable to determine/ unknown/missing }\end{array}$ & $\begin{array}{l}225(86.9) \\
15(5.79) \\
19(7.34)\end{array}$ & $\begin{array}{l}1158(90.0) \\
55(4.28) \\
73(5.68)\end{array}$ & $\begin{array}{l}140(89.7) \\
3(1.92) \\
13(8.33)\end{array}$ & $\begin{array}{l}35(92.1) \\
1(2.63) \\
2(5.26)\end{array}$ & 0.24 & $0.05(0.00-0.09)$ \\
\hline $\begin{array}{l}\text { Decidual vessels, atheroma } \\
\text { Not seen } \\
\text { Present } \\
\text { Unable to determine/ unknown/missing }\end{array}$ & $\begin{array}{l}234(90.3) \\
5(1.93) \\
20(7.72)\end{array}$ & $\begin{array}{l}1194(92.8) \\
15(1.17) \\
77(5.99)\end{array}$ & $\begin{array}{l}142(91.0) \\
1(0.64) \\
13(8.33)\end{array}$ & $\begin{array}{l}36(94.7) \\
0 \\
2(5.26)\end{array}$ & 0.49 & $0.04(0.00-0.07)$ \\
\hline $\begin{array}{l}\text { Syncytium, nuclear clumping } \\
\text { Normal } \\
\text { Less than normal for term placenta } \\
\text { Excessive for term placenta } \\
\text { Excessive in parts } \\
\text { Unable to determine/unknown/missing }\end{array}$ & $\begin{array}{l}183(70.7) \\
52(20.1) \\
10(3.86) \\
0 \\
14(5.41)\end{array}$ & $\begin{array}{l}955(74.3) \\
256(19.9) \\
42(3.27) \\
0 \\
33(2.57)\end{array}$ & $\begin{array}{l}115(73.7) \\
32(20.5) \\
6(3.85) \\
0 \\
3(1.92)\end{array}$ & $\begin{array}{l}28(73.7) \\
7(18.4) \\
1(2.63) \\
0 \\
2(5.26)\end{array}$ & 1.0 & $0.01(0.02-0.07)$ \\
\hline MVM summary score & $0(0,0)$ & $0(0,0)$ & $0(0,0.5)$ & $0(0,0.25)$ & 0.79 & $0.004(-0.05$ to 0.05$)$ \\
\hline Terminal villi & & & & & & \\
\hline $\begin{array}{l}\text { Langhans' layer } \\
\text { Not seen } \\
\text { Present } \\
\text { Unknown/missing } \\
\end{array}$ & $\begin{array}{l}234(90.3) \\
11(4.25) \\
14(5.41) \\
\end{array}$ & $\begin{array}{l}1217(94.6) \\
36(2.80) \\
33(2.57)\end{array}$ & $\begin{array}{l}147(94.2) \\
6(3.85) \\
3(1.93) \\
\end{array}$ & $\begin{array}{l}36(94.7) \\
0 \\
2(5.26) \\
\end{array}$ & 0.24 & $0.04(0.00-0.08)$ \\
\hline $\begin{array}{l}\text { Hofbauer cells } \\
\text { Few } \\
\text { Many } \\
\text { Unknown/missing }\end{array}$ & $\begin{array}{l}227(87.6) \\
18(6.95) \\
14(5.41)\end{array}$ & $\begin{array}{l}1176(91.4) \\
77(5.99) \\
33(2.57)\end{array}$ & $\begin{array}{l}136(87.2) \\
17(10.9) \\
153(1.92)\end{array}$ & $\begin{array}{l}35(92.1) \\
1(2.63) \\
2(5.26)\end{array}$ & 0.11 & $0.06(0.00-0.10)$ \\
\hline $\begin{array}{l}\text { Stromal fibrosis } \\
\text { Not seen } \\
\text { Present } \\
\text { Unable to determine/unknown/missing }\end{array}$ & $\begin{array}{l}231(89.2) \\
14(5.41) \\
14(5.41)\end{array}$ & $\begin{array}{l}1200(93.3) \\
53(4.12) \\
33(2.57)\end{array}$ & $\begin{array}{l}143(91.7) \\
9(5.77) \\
4(2.56)\end{array}$ & $\begin{array}{l}35(92.1) \\
1(2.63) \\
2(5.26)\end{array}$ & 0.59 & $0.03(0.00-0.07)$ \\
\hline $\begin{array}{l}\text { Pathological edema } \\
\text { Not seen } \\
\text { Present } \\
\text { Unable to determine/unknown/missing }\end{array}$ & $\begin{array}{l}230(88.8) \\
15(5.79) \\
14(5.41)\end{array}$ & $\begin{array}{l}1199(93.2) \\
53(4.12) \\
34(2.64)\end{array}$ & $\begin{array}{l}145(92.9) \\
8(5.13) \\
3(1.92)\end{array}$ & $\begin{array}{l}35(92.1) \\
1(2.63) \\
2(5.26)\end{array}$ & 0.57 & $0.04(0.00-0.07)$ \\
\hline Intervillous space & & & & & & \\
\hline $\begin{array}{l}\text { Apparent maturity of placenta } \\
\text { Under } 20 \text { weeks } \\
20-27 \text { weeks } \\
28-36 \text { weeks } \\
37 \text { weeks or over } \\
\text { Unable to determine/unknown/missing }\end{array}$ & $\begin{array}{l}0 \\
11(4.24) \\
57(22.0) \\
177(68.3) \\
14(5.41)\end{array}$ & $\begin{array}{l}4(0.31) \\
33(2.57) \\
260(20.2) \\
952(74.0) \\
37(2.88)\end{array}$ & $\begin{array}{l}0 \\
6(3.85) \\
27(17.3) \\
119(76.3) \\
4(2.56)\end{array}$ & $\begin{array}{l}0 \\
2(5.26) \\
6(15.8) \\
28(73.7) \\
2(5.26)\end{array}$ & 0.55 & $0.04(0.00-0.05)$ \\
\hline Immaturity summary score & $2(2,2)$ & $2(2,2)$ & $2(2,2)$ & $2(2,2)$ & 0.80 & $0.0008(-0.05$ to 0.05$)$ \\
\hline
\end{tabular}

Data are median (IQR; Kruskal-Wallis/Wilcoxon test for non-parametric data with Steel-Dwass post-hoc) for continuous variables or n (\%) (Likelihood Ratio Chi Square test) for categorical variables. $* \mathrm{p}<0.05$. Effect sizes are Wilcoxon test effect size $(\mathrm{r})(95 \% \mathrm{CI})$ for continuous variables, or Cramer's $\mathrm{V}(95 \% \mathrm{CI})$ for categorical variables. $\mathrm{UW}=$ underweight. $\mathrm{NW}=$ normal weight. OW $=$ overweight. $\mathrm{OB}=$ obese. 
Table 2. Multivariable analyses for associations between maternal prepregnancy BMI (continuous) and microscopic placental pathologies in preterm pregnancies, N=1739.

\begin{tabular}{|c|c|c|c|c|c|c|}
\hline \multirow[t]{2}{*}{ Placental pathology } & \multicolumn{2}{|c|}{ Unadjusted model } & \multicolumn{4}{|c|}{ Adjusted model } \\
\hline & $\beta(95 \% \mathrm{CI})$ & OR $(95 \% \mathrm{CI})$ & p-value & $\beta(95 \% \mathrm{CI})$ & OR $(95 \% \mathrm{CI})$ & p-value \\
\hline \multicolumn{7}{|l|}{ Cord neutrophil infiltration } \\
\hline $\begin{array}{l}\text { Umbilical vein } \\
\text { Not seen } \\
\text { Any degree of infiltration }\end{array}$ & $0.07(0.03-0.12)$ & $1.08(1.03-1.12)$ & $* * *$ & $0.06(0.01-0.11)$ & $1.06(1.01-1.12)$ & $* *$ \\
\hline $\begin{array}{l}\text { Umbilical artery } \\
\text { Not seen } \\
\text { Any degree of infiltration }\end{array}$ & $0.05(-0.003$ to 0.10$)$ & $1.05(1.00-1.11)$ & NS & $0.04(-0.02$ to 0.10$)$ & $1.04(0.98-1.10)$ & NS \\
\hline $\begin{array}{l}\text { Umbilical cord substance } \\
\text { Not seen } \\
\text { Any degree of infiltration }\end{array}$ & $0.08(0.03-0.13)$ & $1.09(1.03-1.14)$ & *** & $0.08(0.02-0.13)$ & $1.08(1.02-1.14)$ & $* *$ \\
\hline Fetal inflammation summary score & $0.04(0.01-0.06)$ & - & $* *$ & $0.03(0.003-0.05)$ & - & $*$ \\
\hline \multicolumn{7}{|l|}{ Membranes neutrophil infiltration } \\
\hline $\begin{array}{l}\text { Membrane roll, amnion } \\
\text { Not seen } \\
\text { Any degree of infiltration }\end{array}$ & $0.05(0.006-0.10)$ & $1.05(1.01-1.10)$ & $*$ & $0.03(-0.02$ to 0.08$)$ & $1.03(0.98-1.09)$ & NS \\
\hline $\begin{array}{l}\text { Membrane roll, chorion } \\
\text { Not seen } \\
\text { Any degree of infiltration }\end{array}$ & $0.03(-0.004$ to 0.07$)$ & $1.03(1.00-1.07)$ & NS & $0.02(-0.02$ to 0.06$)$ & $1.02(0.98-1,06)$ & NS \\
\hline $\begin{array}{l}\text { Amnion of placenta } \\
\text { Not seen } \\
\text { Any degree of infiltration }\end{array}$ & $0.03(-0.02$ to 0.09$)$ & $1.04(0.98-1.09)$ & NS & $0.02(-0.04$ to 0.07$)$ & $1.02(0.96-1.08)$ & NS \\
\hline $\begin{array}{l}\text { Placental surface, chorion } \\
\text { Not seen } \\
\text { Any degree of infiltration }\end{array}$ & $0.03(-0.01$ to 0.07$)$ & $1.03(0.99-1.07)$ & NS & $0.01(-0.03$ to 0.05$)$ & $1.01(0.97-1.06)$ & NS \\
\hline Maternal inflammation sum score & $0.04(0.0006-0.08)$ & - & $*$ & $0.02(-0.02$ to 0.07$)$ & - & NS \\
\hline \multicolumn{7}{|l|}{ Decidua } \\
\hline $\begin{array}{l}\text { Decidual vessels, fibrinoid } \\
\text { Not seen } \\
\text { Present }\end{array}$ & $-0.03(-0.11$ to 0.04$)$ & $0.97(0.89-1.05)$ & NS & $-0.05(-0.13$ to 0.04$)$ & $0.96(0.88-1.04)$ & NS \\
\hline $\begin{array}{l}\text { Decidual vessels, atheroma } \\
\text { Not seen } \\
\text { Present }\end{array}$ & $-0.10(-0.26$ to 0.07$)$ & $0.91(0.77-1.07)$ & NS & $-0.12(-0.31$ to 0.07$)$ & $0.89(0.73-1.07)$ & NS \\
\hline $\begin{array}{l}\text { Syncytium, nuclear clumping } \\
\text { Normal/ < normal for term } \\
\text { Excessive/in parts for term }\end{array}$ & $0.01(-0.10$ to 0.07$)$ & $0.99(0.91-1.07)$ & NS & $-0.03(-0.12$ to 0.06$)$ & $0.97(0.88-1.06)$ & NS \\
\hline Maternal vascular malperfusion & $0.006(-0.001$ to 0.01$)$ & - & NS & $0.005(-0.002$ to 0.01$)$ & - & NS \\
\hline \multicolumn{7}{|l|}{ Terminal villi } \\
\hline $\begin{array}{l}\text { Langhans' layer } \\
\text { Not seen } \\
\text { Present }\end{array}$ & $-0.02(-0.11$ to 0.07$)$ & $0.98(0.89-1.07)$ & NS & $-0.04(-0.14$ to 0.06$)$ & $0.96(0.87-1.07)$ & NS \\
\hline $\begin{array}{l}\text { Hofbauer cells } \\
\text { Few } \\
\text { Many }\end{array}$ & $0.03(-0.03$ to 0.09$)$ & $1.03(0.97-1.09)$ & NS & $0.02(-0.04$ to 0.08$)$ & $1.02(0.96-1.09)$ & NS \\
\hline
\end{tabular}




\begin{tabular}{|c|c|c|c|c|c|c|}
\hline $\begin{array}{l}\text { Stromal fibrosis } \\
\text { Not seen } \\
\text { Present }\end{array}$ & $0.02(-0.05$ to 0.09$)$ & $1.02(0.95-1.09)$ & NS & $-0.004(-0.08$ to 0.07$)$ & $1.00(0.93-1.07)$ & NS \\
\hline $\begin{array}{l}\text { Pathological edema } \\
\text { Not seen } \\
\text { Present }\end{array}$ & $0.01(-0.06$ to 0.08$)$ & $1.01(0.94-1.08)$ & NS & $0.02(-0.06$ to 0.09$)$ & $1.02(0.94-1.10)$ & NS \\
\hline \multicolumn{7}{|l|}{ Intervillous space } \\
\hline $\begin{array}{l}\text { Apparent maturity of placenta } \\
\text { Appropriate mature } \\
\text { Hyper-mature }\end{array}$ & $0.02(-0.02$ to 0.05$)$ & $1.02(0.98-1.05)$ & NS & $0.02(-0.02$ to 0.06$)$ & $1.02(0.98-1.07)$ & NS \\
\hline Immaturity summary score & 0.0006 (-0.006 to 0.007$)$ & - & NS & $0.002(-0.005$ to 0.009$)$ & - & NS \\
\hline
\end{tabular}

Data are $\beta(95 \% \mathrm{CI})$ and unit odds ratios $(95 \% \mathrm{CI})$ for presence of pathology from Logistic regression models with p-value from Likelihood Ratio Chi Square test for categorical variables, or $\beta(95 \% \mathrm{CI})$ with p-value from Standard Least Squares models for continuous variables. The adjusted model includes fetal sex, maternal race, maternal age, smoking history, maternal education, socioeconomic status, gestational weight gain, and maternal diabetes mellitus status. NS (not significant): $\mathrm{p}>0.05 ;{ }^{*} \mathrm{p} \leq 0.05 ; * * \mathrm{p} \leq 0.01 ; * * * \mathrm{p} \leq 0.001 ; * * * \mathrm{p} \leq 0.0001$. 
Table 3. Associations between maternal prepregnancy BMI and microscopic placental pathologies in term pregnancies, N=10,145.

\begin{tabular}{|c|c|c|c|c|c|c|}
\hline \multirow{2}{*}{ Placental pathology } & \multicolumn{4}{|c|}{ Prepregnancy BMI } & \multirow[b]{2}{*}{ p-value } & \multirow[b]{2}{*}{ Effect size } \\
\hline & $\begin{array}{l}\text { UW } \\
(\mathrm{n}=1201)\end{array}$ & $\begin{array}{l}\text { NW } \\
(\mathrm{n}=8010)\end{array}$ & $\begin{array}{l}\text { OW } \\
(\mathrm{n}=922)\end{array}$ & $\begin{array}{l}\text { OB } \\
(\mathrm{n}=282)\end{array}$ & & \\
\hline \multicolumn{7}{|l|}{ Cord } \\
\hline Neutrophil infiltration, umbilical vein & & & & & 0.001 & $0.03(0.01-0.04)$ \\
\hline Not seen & 1065 (88.7) & $6957(86.9)$ & $794(86.1)$ & $237(84.0)$ & & \\
\hline Slight & $84(6.99)$ & $601(7.50)$ & $54(5.86)$ & $22(7.80)$ & & \\
\hline Moderate & 0 & $16(0.20)$ & 0 & 0 & & \\
\hline Marked & $24(2.0)$ & $197(2.46)$ & $35(3.80)$ & $16(5.67)$ & & \\
\hline Unknown/missing & $28(2.33)$ & $239(2.98)$ & $39(4.23)$ & $7(2.48)$ & & \\
\hline Neutrophil infiltration, umbilical artery & & & & & 0.04 & $0.03(0.009-0.03)$ \\
\hline Not seen & $1141(95.0)$ & $7552(94.3)$ & $850(92.2)$ & $260(92.2)$ & & \\
\hline Slight & $20(1.67)$ & $141(1.76)$ & $17(1.84)$ & $5(1.77)$ & & \\
\hline Moderate & 0 & $3(0.04)$ & 0 & 0 & & \\
\hline Marked & $12(1.0)$ & $75(0.94)$ & $17(1.84)$ & $10(3.55)$ & & \\
\hline Unknown/missing & $28(2.33)$ & $239(2.98)$ & $38(4.12)$ & $7(2.48)$ & & \\
\hline Neutrophil infiltration, umbilical cord substance & & & & & 0.05 & $0.03(0.007-0.03)$ \\
\hline Not seen & $1130(94.1)$ & $7431(92.8)$ & $835(90.6)$ & $253(89.7)$ & & \\
\hline Slight & $34(2.83)$ & $264(3.30)$ & $37(4.01)$ & $13(4.61)$ & & \\
\hline Moderate & 0 & $5(0.06)$ & $1(0.11)$ & 0 & & \\
\hline Marked & $9(0.75)$ & $71(0.89)$ & $11(1.19)$ & $9(3.19)$ & & \\
\hline Unknown/missing & $28(2.33)$ & $239(2.98)$ & $38(4.12)$ & $7(2.48)$ & & \\
\hline Fetal inflammation summary score & $0(0,0)$ & $0(0,0)$ & $0(0,0)$ & $0(0,0)$ & 0.07 & $0.01(-0.008$ to 0.03$)$ \\
\hline \multicolumn{7}{|l|}{ Membranes } \\
\hline Neutrophil infiltration, membrane roll, amnion & & & & & 0.002 & $0.03(0.01-0.04)$ \\
\hline Not seen & $1028(85.6)$ & $6728(84.0)$ & $739(80.2)$ & $224(79.4)$ & & \\
\hline Slight & $62(5.16)$ & $434(5.42)$ & $71(7.70)$ & $25(8.87)$ & & \\
\hline Moderate & 0 & $5(0.06)$ & 0 & $1(0.35)$ & & \\
\hline Marked & $16(1.33)$ & $156(1.95)$ & $25(2.71)$ & $10(3.55)$ & & \\
\hline Unknown/missing & $95(7.91)$ & $687(8.58)$ & $87(9.44)$ & $22(7.80)$ & & \\
\hline Neutrophil infiltration, membrane roll, chorion & & & & & 0.003 & $0.03(0.01-0.04)$ \\
\hline Not seen & $1032(85.9)$ & $6710(83.8)$ & $750(81.34)$ & $216(76.6)$ & & \\
\hline Slight & $97(8.08)$ & $761(9.50)$ & $88(9.54)$ & $41(14.5)$ & & \\
\hline Moderate & $2(0.17)$ & $9(0.11)$ & 0 & 0 & & \\
\hline Marked & $39(3.25)$ & $286(3.57)$ & $46(4.99)$ & $19(6.74)$ & & \\
\hline Unknown/missing & $31(2.58)$ & $244(3.05)$ & $38(4.12)$ & $6(2.13)$ & & \\
\hline Neutrophil infiltration, placental surface, amnion & & & & & $\mathbf{0 . 0 3}$ & $0.03(0.009-0.04)$ \\
\hline Not seen & $1011(84.2)$ & $6683(83.43)$ & $747(81.0)$ & $237(84.0)$ & & \\
\hline Slight & $44(3.66)$ & $288(3.60)$ & $47(5.10)$ & $13(4.61)$ & & \\
\hline Moderate & $1(0.08)$ & $2(0.02)$ & $1(0.11)$ & $1(0.35)$ & & \\
\hline Marked & $9(0.75)$ & $95(1.19)$ & $17(1.84)$ & $8(2.84)$ & & \\
\hline Unknown/missing & $136(11.32)$ & $942(11.8)$ & $110(11.9)$ & $23(8.16)$ & & \\
\hline Neutrophil infiltration, placental surface, chorion & & & & & 0.04 & $0.03(0.005-0.03)$ \\
\hline Not seen & 1070 (89.1) & $6976(87.1)$ & 773 (83.8) & $239(84.8)$ & & \\
\hline Slight & $85(7.08)$ & $620(7.74)$ & $83(9.00)$ & $22(7.80)$ & & \\
\hline Moderate & $1(0.08)$ & $6(0.07)$ & $1(0.11)$ & $1(0.35)$ & & \\
\hline Marked & $19(1.58)$ & $185(2.31)$ & $29(3.15)$ & $14(4.96)$ & & \\
\hline Unknown/missing & $26(2.16)$ & $223(2.78)$ & $36(3.90)$ & $6(2.13)$ & & \\
\hline Maternal inflammation summary score & $0(0,0)^{\mathrm{A}}$ & $0(0,0)^{\mathrm{AB}}$ & $0(0,0)^{\mathrm{AB}}$ & $0(0,1)^{\mathrm{B}}$ & 0.009 & $0.008(-0.01$ to 0.03$)$ \\
\hline
\end{tabular}




\begin{tabular}{|c|c|c|c|c|c|c|}
\hline \multicolumn{7}{|l|}{ Decidua } \\
\hline Decidual vessels, fibrinoid & & & & & 0.19 & $0.02(0.00-0.04)$ \\
\hline Not seen & $1106(92.1)$ & 7405 (92.4) & $849(92.1)$ & $259(91.8)$ & & \\
\hline Present & $44(3.66)$ & $226(2.82)$ & $19(2.06)$ & $7(2.48)$ & & \\
\hline Unable to determine/ unknown/missing & $51(4.25)$ & $379(4.73)$ & $36(3.90)$ & $16(5.67)$ & & \\
\hline Decidual vessels, atheroma & & & & & 0.25 & $0.02(0.00-0.04)$ \\
\hline Not seen & $1127(93.8)$ & 7521 (93.9) & $854(92.6)$ & $262(92.9)$ & & \\
\hline Present & $21(1.75)$ & $83(1.04)$ & $10(1.08)$ & $3(1.06)$ & & \\
\hline Unable to determine/ unknown/missing & $53(4.41)$ & $406(5.07)$ & $58(6.29)$ & $17(6.03)$ & & \\
\hline Syncytium, nuclear clumping & & & & & 0.61 & $0.02(0.00-0.02)$ \\
\hline Normal & $1046(87.1)$ & $6962(86.9)$ & $799(86.7)$ & $253(89.7)$ & & \\
\hline Less than normal for term placenta & $97(8.08)$ & $556(6.94)$ & $60(6.51)$ & $13(4.61)$ & & \\
\hline Excessive for term placenta & $29(2.41)$ & $237(2.96)$ & $22(2.39)$ & $9(3.19)$ & & \\
\hline Excessive in parts & $5(0.41)$ & $41(0.51)$ & $5(0.54)$ & $1(0.35)$ & & \\
\hline Unable to determine/unknown/missing & $24(2.00)$ & $214(2.67)$ & $36(3.90)$ & $6(2.13)$ & & \\
\hline Maternal vascular malperfusion summary score & $0(0,1)$ & $0(0,1)$ & $0(0,1)$ & $0(0,1)$ & 0.07 & $0.02(-0.004$ to 0.04$)$ \\
\hline \multicolumn{7}{|l|}{ Terminal villi } \\
\hline Langhans' layer & & & & & 0.40 & $0.02(0.00-0.03)$ \\
\hline Not seen & $1173(97.7)$ & $7753(96.8)$ & $879(95.3)$ & $273(96.8)$ & & \\
\hline Present & $4(0.33)$ & $46(0.57)$ & $7(0.76)$ & $3(1.06)$ & & \\
\hline Unknown/missing & $24(2.00)$ & $211(2.63)$ & $36(3.90)$ & $6(2.13)$ & & \\
\hline Hofbauer cells & & & & & 0.90 & $0.007(0.00-0.02)$ \\
\hline Few & 1155 (96.2) & 7660 (95.6) & 872 (94.6) & $271(96.1)$ & & \\
\hline Many & $22(1.83)$ & $140(1.75)$ & $13(1.41)$ & $5(1.77)$ & & \\
\hline Unknown/missing & $24(2.00)$ & $210(2.62)$ & $37(4.01)$ & $6(2.13)$ & & \\
\hline Stromal fibrosis & & & & & 0.23 & $0.02(0.00-0.04)$ \\
\hline Not seen & $1135(94.5)$ & 7487 (93.5) & $861(93.4)$ & $263(93.3)$ & & \\
\hline Present & $41(3.41)$ & $313(3.91)$ & $25(2.71)$ & $13(4.61)$ & & \\
\hline Unable to determine/unknown/missing & $25(2.08)$ & $210(2.62)$ & $36(3.90)$ & $6(2.13)$ & & \\
\hline Pathological edema & & & & & 0.11 & $0.02(0.00-0.04)$ \\
\hline Not seen & 1135 (94.5) & $7541(94.1)$ & $857(93.0)$ & $273(96.8)$ & & \\
\hline Present & $42(3.50)$ & $258(3.22)$ & $29(3.14)$ & $3(1.06)$ & & \\
\hline Unable to determine/unknown/missing & $24(2.00)$ & $211(2.63)$ & $36(3.90)$ & $6(2.13)$ & & \\
\hline \multicolumn{7}{|l|}{ Intervillous space } \\
\hline Apparent maturity of placenta & & & & & 0.70 & $0.01(0.00-0.01)$ \\
\hline Under 20 weeks & $1(0.08)$ & $6(0.07)$ & 0 & 0 & & \\
\hline 20-27 weeks & $4(0.33)$ & $19(0.24)$ & $1(0.11)$ & 0 & & \\
\hline 28-36 weeks & $92(7.66)$ & $543(6.78)$ & $55(5.97)$ & $19(6.74)$ & & \\
\hline 37 weeks or over & $1078(89.8)$ & $7225(90.2)$ & $830(90.0)$ & $257(91.1)$ & & \\
\hline Unable to determine/unknown/missing & $26(2.16)$ & $217(2.71)$ & $36(3.90)$ & $6(2.16)$ & & \\
\hline Immaturity summary score & $2(2,2)$ & $2(2,2)$ & $2(2,2)$ & $2(2,2)$ & 0.38 & $-0.01(-0.03$ to 0.008$)$ \\
\hline
\end{tabular}

Data are median (IQR; Kruskal-Wallis/Wilcoxon test for non-parametric data with Steel-Dwass post-hoc) for continuous variables or $\mathrm{n}$ (\%) (Likelihood Ratio Chi Square test) for categorical variables. $* \mathrm{p}<0.05$. Effect sizes are Wilcoxon test effect size $(\mathrm{r})(95 \% \mathrm{CI})$ for continuous variables, or Cramer's V $(95 \% \mathrm{CI})$ for categorical variables. UW $=$ underweight. NW $=$ normal weight. OW $=$ overweight $\mathrm{OB}=$ obese. 
Table 4. Multivariable analyses for associations between maternal prepregnancy BMI (continuous) and microscopic placental pathologies in term pregnancies, N=10,145.

\begin{tabular}{|c|c|c|c|c|c|c|}
\hline \multirow[t]{2}{*}{ Placental pathology } & \multicolumn{2}{|c|}{ Unadjusted model } & \multicolumn{4}{|c|}{ Adjusted model } \\
\hline & $\beta(95 \% \mathrm{CI})$ & OR $(95 \% \mathrm{CI})$ & p-value & $\beta(95 \% \mathrm{CI})$ & OR $(95 \% \mathrm{CI})$ & p-value \\
\hline \multicolumn{7}{|l|}{ Cord neutrophil infiltration } \\
\hline $\begin{array}{l}\text { Umbilical vein } \\
\text { Not seen } \\
\text { Any degree of infiltration }\end{array}$ & $0.02(0.004-0.04)$ & $1.02(1.00-1.04)$ & $*$ & $0.03(0.007-0.05)$ & $1.03(1.01-1.05)$ & $* *$ \\
\hline $\begin{array}{l}\text { Umbilical artery } \\
\text { Not seen } \\
\text { Any degree of infiltration }\end{array}$ & $0.05(0.02-0.08)$ & $1.05(1.02-1.08)$ & $* *$ & $0.04(0.004-0.07)$ & $1.04(1.00-1.07)$ & $*$ \\
\hline $\begin{array}{l}\text { Umbilical cord substance } \\
\text { Not seen } \\
\text { Any degree of infiltration }\end{array}$ & $0.04(0.02-0.07)$ & $1.04(1.02-1.07)$ & $* * *$ & $0.04(0.009-0.06)$ & $1.04(1.01-1.07)$ & $* *$ \\
\hline Fetal inflammation score & $0.01(0.008-0.02)$ & - & $* * * *$ & $0.01(0.007-0.02)$ & - & $* * * *$ \\
\hline \multicolumn{7}{|c|}{ Membranes and neutrophil infiltration } \\
\hline $\begin{array}{l}\text { Membrane roll, amnion } \\
\text { Not seen } \\
\text { Any degree of infiltration }\end{array}$ & $0.06(0.04-0.07)$ & $1.06(1.04-1.08)$ & $* * * *$ & $0.05(0.03-0.08)$ & $1.06(1.03-1.08)$ & $* * * *$ \\
\hline $\begin{array}{l}\text { Membrane roll, chorion } \\
\text { Not seen } \\
\text { Any degree of infiltration }\end{array}$ & $0.05(0.03-0.06)$ & $1.05(1.03-1.07)$ & $* * * *$ & $0.04(0.02-0.06)$ & $1.04(1.02-1.06)$ & $* * * *$ \\
\hline $\begin{array}{l}\text { Placental surface, amnion } \\
\text { Not seen } \\
\text { Any degree of infiltration }\end{array}$ & $0.04(0.01-0.06)$ & $1.04(1.01-1.06)$ & $* *$ & $0.04(0.01-0.06)$ & $1.04(1.01-1.07)$ & *** \\
\hline $\begin{array}{l}\text { Placental surface, chorion } \\
\text { Not seen } \\
\text { Any degree of infiltration }\end{array}$ & $0.04(0.02-0.06)$ & $1.04(1.02-1.06)$ & $* * * *$ & $0.04(0.02-0.06)$ & $1.04(1.02-1.06)$ & $* * *$ \\
\hline Maternal inflammation score & $0.03(0.02-0.04)$ & - & $* * * *$ & $0.03(0.02-0.04)$ & - & $* * * *$ \\
\hline \multicolumn{7}{|l|}{ Decidua } \\
\hline $\begin{array}{l}\text { Decidual vessels, fibrinoid } \\
\text { Not seen } \\
\text { Present }\end{array}$ & $-0.03(-0.07$ to 0.01$)$ & $0.97(0.94-1.01)$ & NS & $-0.02(-0.06$ to 0.02$)$ & $0.98(0.94-1.02)$ & NS \\
\hline $\begin{array}{l}\text { Decidual vessels, atheroma } \\
\text { Not seen } \\
\text { Present }\end{array}$ & $-0.05(-0.12$ to 0.01$)$ & $0.95(0.89-1.01)$ & NS & $-0.06(-0.13$ to 0.004$)$ & $0.94(0.88-1.00)$ & NS \\
\hline $\begin{array}{l}\text { Syncytium, nuclear clumping } \\
\text { Normal/ < normal for term } \\
\text { Excessive/in parts for term }\end{array}$ & $0.002(-0.03$ to 0.03$)$ & $1.00(0.71-1.03)$ & NS & $0.001(-0.03$ to 0.04$)$ & $1.00(0.97-1.04)$ & NS \\
\hline Maternal vascular malperfusion & $0.005(0.002-0.008)$ & - & $* *$ & $0.007(0.004-0.01)$ & - & $* * * *$ \\
\hline \multicolumn{7}{|l|}{ Terminal villi } \\
\hline $\begin{array}{l}\text { Langhans' layer } \\
\text { Not seen } \\
\text { Present }\end{array}$ & $0.007(-0.07$ to 0.08$)$ & $1.01(0.93-1.09)$ & NS & $-0.01(-0.09$ to 0.07$)$ & $0.99(0.91-1.07)$ & NS \\
\hline $\begin{array}{l}\text { Hofbauer cells } \\
\text { Few } \\
\text { Many }\end{array}$ & $-0.03(-0.08$ to 0.02$)$ & $0.97(0.92-1.02)$ & NS & $-0.04(-0.09$ to 0.009$)$ & $0.96(0.91-1.01)$ & NS \\
\hline
\end{tabular}




\begin{tabular}{|c|c|c|c|c|c|c|}
\hline $\begin{array}{l}\text { Stromal fibrosis } \\
\text { Not seen } \\
\text { Present }\end{array}$ & $-0.006(-0.04$ to 0.03$)$ & $0.99(0.96-1.03)$ & NS & $0.002(-0.03$ to 0.04$)$ & $1.00(0.97-1.04)$ & NS \\
\hline $\begin{array}{l}\text { Pathological edema } \\
\text { Not seen } \\
\text { Present } \\
\end{array}$ & $-0.03(-0.07$ to 0.004$)$ & $0.97(0.93-1.00)$ & NS & $-0.01(-0.05$ to 0.03$)$ & $0.99(0.95-1.03)$ & NS \\
\hline \multicolumn{7}{|l|}{ Intervillous space } \\
\hline $\begin{array}{l}\text { Apparent maturity of placenta } \\
\text { Appropriate mature } \\
\text { Immature }\end{array}$ & $-0.02(-0.05$ to 0.003$)$ & $0.98(0.96-1.00)$ & NS & $-0.60(-1.37$ to 0.18$)$ & $0.94(0.92-0.97)$ & $* * * *$ \\
\hline Immaturity score & $-0.0003(-0.002$ to 0.002$)$ & - & NS & $-0.0009(-0.003$ to 0.001$)$ & - & NS \\
\hline
\end{tabular}

Data are $\beta(95 \% \mathrm{CI})$ and unit odds ratios $(95 \% \mathrm{CI})$ for presence of pathology from Logistic regression models with p-value from Likelihood Ratio Chi Square test for categorical variables, or $\beta(95 \% \mathrm{CI})$ with p-value and from Standard Least Squares models for continuous variables. The adjusted model includes fetal sex, maternal race, maternal age, smoking history, maternal education, socioeconomic status, gestational weight gain, and maternal diabetes mellitus status. NS (not significant): $\mathrm{p}>0.05 ;{ }^{*} \mathrm{p} \leq 0.05 ; * * \mathrm{p} \leq 0.01 ;{ }^{* * *} \mathrm{p} \leq 0.001 ;{ }^{* * * *} \mathrm{p} \leq 0.0001$. 
Table 5. Associations between fetal sex and microscopic placental pathologies in preterm $(\mathrm{N}=1739)$ and term $(\mathrm{N}=10,415)$ pregnancies.

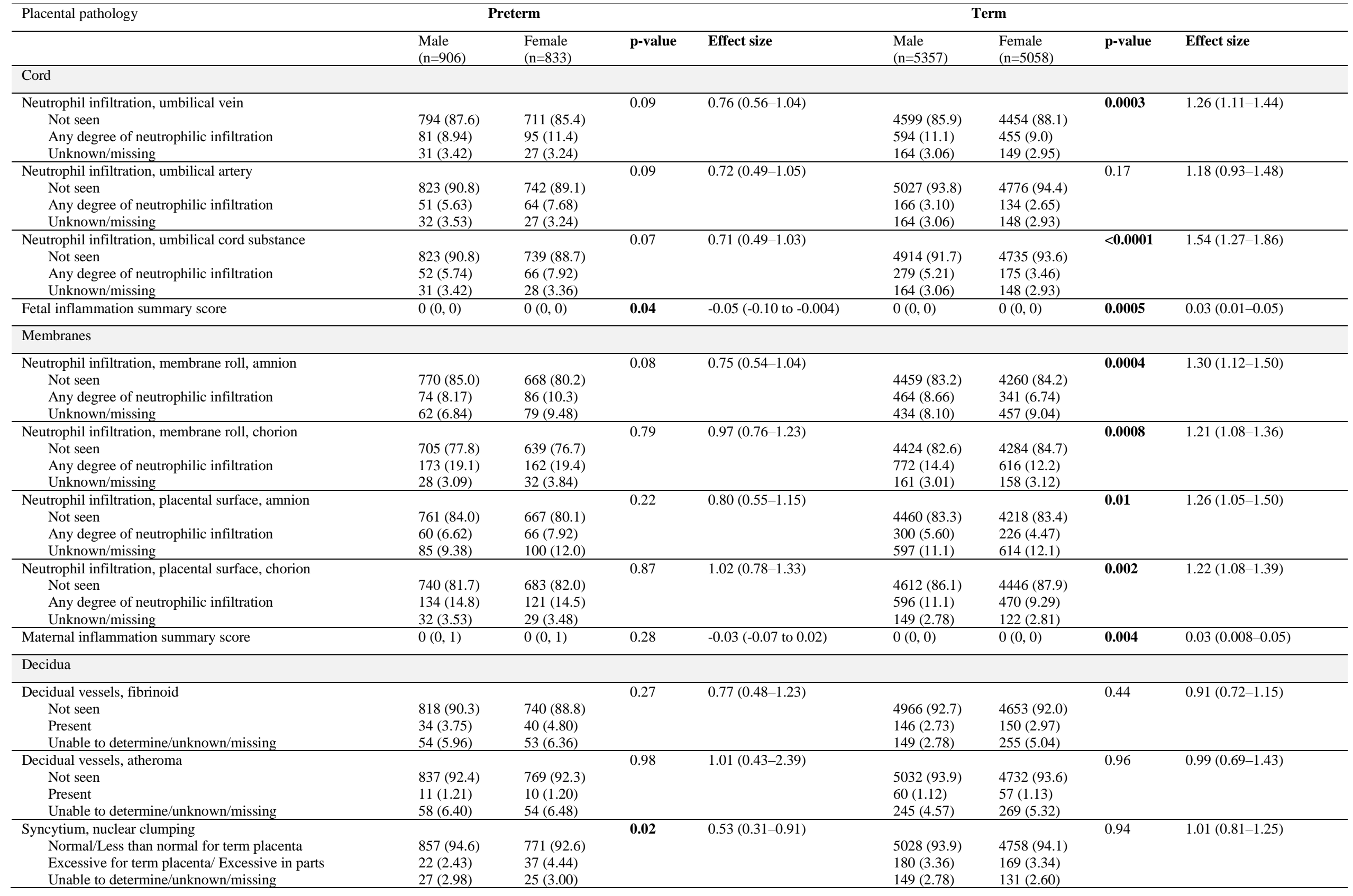

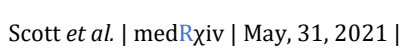




\begin{tabular}{|c|c|c|c|c|c|c|c|c|}
\hline Maternal vascular malperfusion summary score & $0(0,0)$ & $0(0,0)$ & 0.89 & $-0.003(-0.05$ to 0.05$)$ & $0(0,1)$ & $0(0,1)$ & $<0.0001$ & $0.04(0.02-0.06)$ \\
\hline \multicolumn{9}{|l|}{ Terminal villi } \\
\hline Langhans' layer & & & 0.47 & $0.82(0.47-1.41)$ & & & 0.76 & $1.08(0.65-1.80)$ \\
\hline Not seen & $854(94.3)$ & $780(93.6)$ & & & $5178(96.7)$ & 4900 (96.9) & & \\
\hline Unknown/missing & $27(2.98)$ & $25(3.00)$ & & & $147(2.74)$ & $130(2.57)$ & & \\
\hline Hofbauer cells & & & 0.23 & $1.26(0.86-1.86)$ & & & 0.94 & $0.99(0.74-1.33)$ \\
\hline Few & $814(89.8)$ & $760(91.2)$ & & & $5119(95.6)$ & 4839 (95.7) & & \\
\hline Many & $65(7.17)$ & $48(5.76)$ & & & $92(1.72)$ & $88(1.74)$ & & \\
\hline Unknown/missing & $27(2.98)$ & $25(3.00)$ & & & $146(2.73)$ & $131(2.59)$ & & \\
\hline Stromal fibrosis & & & 0.17 & $1.39(0.87-2.21)$ & & & 0.09 & $0.84(0.69-1.03)$ \\
\hline Not seen & $832(91.8)$ & $777(93.3)$ & & & $5026(93.8)$ & $4720(93.3)$ & & \\
\hline Present & $46(5.08)$ & $31(3.72)$ & & & $185(3.45)$ & $207(4.09)$ & & \\
\hline Unable to determine/unknown/missing & $28(3.09)$ & $25(3.00)$ & & & $146(2.73)$ & $131(2.59)$ & & \\
\hline Pathological edema & & & 0.17 & $1.38(0.87-2.20)$ & & & 0.11 & $1.20(0.96-1.49)$ \\
\hline Not seen & $833(91.9)$ & $776(93.2)$ & & & $5025(93.8)$ & $4781(94.5)$ & & \\
\hline Present & $46(5.08)$ & $31(3.72)$ & & & $185(3.45)$ & $147(2.91)$ & & \\
\hline Unable to determine/unknown/missing & $27(2.98)$ & $26(3.12)$ & & & $147(2.74)$ & $130(2.57)$ & & \\
\hline \multicolumn{9}{|l|}{ Intervillous space } \\
\hline Apparent maturity of placenta & & & 0.27 & - & & & 0.17 & - \\
\hline 20-27 weeks & $21(2.32)$ & $31(3.72)$ & & & $14(0.26)$ & $10(0.20)$ & & \\
\hline 28-36 weeks & $192(21.2)$ & $158(19.0)$ & & & $371(6.93)$ & $338(6.68)$ & & \\
\hline 37 weeks or over & $663(73.2)$ & $613(73.6)$ & & & $4819(90.0)$ & $4571(90.4)$ & & \\
\hline Unable to determine/unknown/missing & $28(3.09)$ & $29(3.48)$ & & & $152(2.84)$ & $133(2.63)$ & & \\
\hline Immaturity summary score & $2(2,2)$ & $2(2,2)$ & 0.45 & $0.02(-0.03$ to 0.06$)$ & $2(2,2)$ & $2(2,2)$ & 0.23 & $0.01(-0.008$ to 0.03$)$ \\
\hline
\end{tabular}

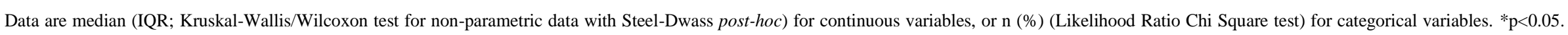
Effect sizes are Wilcoxon test effect size (r) $(95 \% \mathrm{CI})$ for continuous variables, or odds ratios $(95 \% \mathrm{CI})$ for presence of pathology in male placentae for categorical variables. 
Table 6. Associations between fetal sex and macroscopic placental pathologies in preterm $(\mathrm{N}=1739)$ and term $(\mathrm{N}=10,415)$ pregnancies.

\begin{tabular}{|c|c|c|c|c|c|c|c|c|}
\hline \multirow[t]{2}{*}{ Placental pathology } & \multicolumn{2}{|c|}{ Preterm } & \multicolumn{6}{|c|}{ Term } \\
\hline & $\begin{array}{l}\text { Male } \\
(\mathrm{n}=906)\end{array}$ & $\begin{array}{l}\text { Female } \\
(\mathrm{n}=833)\end{array}$ & p-value & Effect size & $\begin{array}{l}\text { Male } \\
(\mathrm{n}=5357)\end{array}$ & $\begin{array}{l}\text { Female } \\
(\mathrm{n}=5058)\end{array}$ & p-value & Effect size \\
\hline \multicolumn{9}{|l|}{ Placenta $^{1}$} \\
\hline Placenta shape & & & 0.64 & - & & & 0.17 & - \\
\hline Non-partite & $804(88.7)$ & $727(87.3)$ & & & 4839 (90.3) & $4553(90.0)$ & & \\
\hline Bipartite & $4(0.44)$ & $1(0.12)$ & & & $65(1.21)$ & $68(1.34)$ & & \\
\hline Tripartite & 0 & 0 & & & 0 & $2(0.04)$ & & \\
\hline Succenturiate & $12(1.32)$ & $11(1.32)$ & & & $61(1.14)$ & $53(1.05)$ & & \\
\hline Membranous placenta & 0 & 0 & & & 0 & $2(0.04)$ & & \\
\hline Crescent shaped & 0 & 0 & & & $2(0.04)$ & 0 & & \\
\hline Irregular shape & $4(0.44)$ & $3(0.36)$ & & & $23(0.43)$ & $23(0.45)$ & & \\
\hline Unknown/missing & $82(9.05)$ & $91(10.9)$ & & & $518(9.67)$ & $357(7.06)$ & & \\
\hline Weight & $382(320,450)$ & $375(320,435)$ & 0.17 & $0.03(-0.02$ to 0.08$)$ & $425(370,485)$ & $420(370,480)$ & 0.06 & $0.02(-0.002$ to 0.04$)$ \\
\hline Largest diameter & $18(17,19)$ & $18(17,19)$ & 0.61 & $0.01(-0.04$ to 0.06$)$ & $19(18,20)$ & $19(18,20)$ & 0.03 & $0.02(0.002-0.04)$ \\
\hline Smallest diameter & $16(14,17)$ & $15(14,17)$ & 0.41 & $0.02(-0.03$ to 0.07$)$ & $16(15,18)$ & $16(15,17)$ & 0.19 & $0.01(-0.006$ to 0.03$)$ \\
\hline Thickness & $20(18,24)$ & $20(18,23)$ & 0.38 & $0.02(-0.02$ to 0.07$)$ & $20(20,25)$ & $20(20,25)$ & 0.53 & $0.006(-0.02$ to 0.03$)$ \\
\hline Birthweight : placenta weight & $7.34(6.52,8.32)$ & $7.16(6.36,7.93)$ & 0.003 & $0.07(0.03-0.12)$ & $7.56(6.78,8.38)$ & $7.36(6.65,8.17)$ & $<0.0001$ & $0.07(0.05-0.09)$ \\
\hline Birthweight : placental largest diameter & $157(140,175)$ & $150(135,166)$ & $<0.0001$ & $0.13(0.09-0.018)$ & $169(155,184)$ & $165(151,179)$ & $<0.0001$ & $0.09(0.07-0.11)$ \\
\hline Birthweight : placental smallest diameter & $183(164,202)$ & $173(156,193)$ & $<0.0001$ & $0.12(0.08-0.17)$ & $197(179,214)$ & $191(175,208)$ & $<0.0001$ & $0.09(0.07-0.11)$ \\
\hline Birthweight : placental thickness & $140(118,162)$ & $133(116,155)$ & 0.003 & $0.07(0.03-0.12)$ & $147(126,170)$ & $143(125,164)$ & $<0.0001$ & $0.06(0.04-0.08)$ \\
\hline \multicolumn{9}{|l|}{ Cord } \\
\hline Cord number of vessels & & & 0.85 & $0.90(0.29-2.79)$ & & & 0.10 & $0.66(0.40-1.09)$ \\
\hline Two & $821(90.6)$ & $736(88.4)$ & & & 4965 (92.7) & $4659(92.1)$ & & \\
\hline Three & $6(0.66)$ & $6(0.72)$ & & & $26(0.49)$ & $37(0.73)$ & & \\
\hline Other/unknown/missing & $79(8.72)$ & $91(10.9)$ & & & $366(6.83)$ & $362(7.16)$ & & \\
\hline Cord edema & & & 0.97 & $1.01(0.62-1.64)$ & & & 0.02 & $1.46(1.07-1.99)$ \\
\hline Not seen & $781(86.2)$ & $700(84.0)$ & & & 4817 (89.9) & $4562(90.2)$ & & \\
\hline Present & $36(3.97)$ & $31(3.72)$ & & & $103(1.92)$ & $67(1.32)$ & & \\
\hline Unknown/missing & $89(9.82)$ & $101(12.1)$ & & & $437(8.16)$ & $429(8.48)$ & & \\
\hline \multicolumn{9}{|l|}{ Membranes and fetal surface } \\
\hline Opacity of membranes & & & 0.97 & $1.01(0.69-1.46)$ & & & 0.45 & $1.06(0.91-1.25)$ \\
\hline Not opaque & $763(84.2)$ & $684(82.1)$ & & & $4648(86.8)$ & 4384 (86.7) & & \\
\hline Any degree of opacity & $64(7.06)$ & $57(6.84)$ & & & $347(6.48)$ & $308(6.09)$ & & \\
\hline Unknown/missing & $79(8.72)$ & $92(11.0)$ & & & $362(6.76)$ & $366(7.24)$ & & \\
\hline Thrombosed fetal vessels & & & 0.93 & $1.05(0.35-3.13)$ & & & 0.73 & $0.88(0.42-1.82)$ \\
\hline Not seen & $821(90.6)$ & 738 (88.6) & & & 4983 (93.0) & 4687 (92.7) & & \\
\hline Present & $7(0.77)$ & $6(0.72)$ & & & $14(0.26)$ & $15(0.30)$ & & \\
\hline Unknown/missing & $78(8.61)$ & $89(10.7)$ & & & $360(6.72)$ & $356(7.04)$ & & \\
\hline \multicolumn{9}{|l|}{ Cut surface } \\
\hline Infarct size & & & 0.89 & $0.98(0.77-1.26)$ & & & $<0.0001$ & $1.22(1.11-1.33)$ \\
\hline Not applicable & $660(72.8)$ & $593(71.2)$ & & & 3546 (66.2) & $3513(69.5)$ & & \\
\hline Any infarcts seen & $164(18.1)$ & $150(18.0)$ & & & $1433(26.8)$ & $1167(23.1)$ & & \\
\hline Unknown/missing & $82(9.05)$ & $90(10.8)$ & & & $378(7.06)$ & $378(7.47)$ & & \\
\hline
\end{tabular}


Cut surface calcification

None

Any calcification seen

Unknown/missing
0.43

$1.08(0.89-1.32)$

414 (49.7)

$380(41.9)$

327 (39.3)

82 (9.05)
$2051(66.2)$

2926 (54.6)

380 (7.09)
$1860(36.8)$

$2819(55.7)$

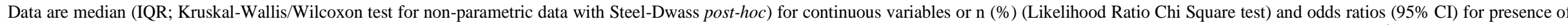

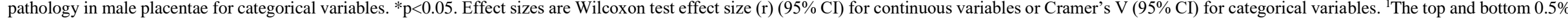

of raw infant and placental anthropometry data (birthweight [g], placental weight [g], placental diameter [cm], and placental thickness [mm]) were excluded to remove biologically implausible data. 
Female preterm placentae had increased syncytium-nuclear clumping $(\mathrm{OR}=0.53$ [0.31-0.91], Table 5), but there were no differences in presence of infarcts or MVM summary score between sexes preterm. Male term placentae had increased MVM ( $\mathrm{r}=0.04$ [0.02-0.06], Table 5) and placental infarcts compared to female placentae $(\mathrm{OR}=1.22$ [1.111.33], Table 6). Infant birthweight to placental weight ratio was also increased in males compared to females at preterm $(\mathrm{r}=0.07$ [0.03-0.12]) and term ( $\mathrm{r}=0.07$ [0.05-0.09], Table 6).

\section{Discussion}

We evaluated the associations between maternal prepregnancy BMI and the prevalence of placental pathologies in preterm and term pregnancies, to better understand the placental mechanisms that may explain poor pregnancy and offspring outcomes in pregnancies complicated by suboptimal maternal BMI. Using data from 12,154 pregnancies from the Collaborative Perinatal Project, we found placental inflammation was increased in pregnancies complicated by maternal obesity, and notably, the inflammatory response was different based on gestational age. Maternal obesity also associated with increased MVM of the placenta, and increased maternal BMI associated with greater odds of having an appropriate mature placenta at term. Placental efficiency was highest in pregnancies where mothers were underweight and in male placentae, and pathologies also differed by fetal sex. Suboptimal maternal BMI thus alters normal gestational tissue development, with likely effects on function.

The inflammatory conditions established by maternal obesity may favour placental inflammation. In support of this, we found that higher maternal BMI associated with increased fetal (e.g. neutrophilic infiltration of the umbilical vein, artery, and cord substance) inflammation at preterm, and maternal (e.g. neutrophilic infiltration of the amnion and chorion membranes, amnion and chorion of the placenta, and opacity of membranes) gestational tissue inflammation at term. While few studies have characterised fetal- and maternal-specific placental inflammation in the context of both maternal BMI and gestational age, at term, maternal inflammation has been documented in pregnancies with obesity, evidenced by increased pro-inflammatory cytokines in the placenta ${ }^{41}$, and increased maternal, but not fetal, inflammatory lesions ${ }^{17,42}$. Other studies have found no differences in placental inflammation by maternal BMI in term pregnancies, however, these cohorts had a small number of cases ${ }^{43}$, ${ }^{44}$. Less is known about the relationships between maternal obesity and specific placental inflammation at preterm, however, maternal obesity has been associated with greater risk of chorioamnionitis leading to preterm birth ${ }^{45}$, and a combination of maternal (defined as inflammation in the chorion, amnion, and decidua) and fetal (inflammation of the umbilical cord and chorionic plate fetal vessels) gestational tissue inflammation has been linked to higher risk of extreme preterm birth than maternal gestational tissue inflammation alone ${ }^{46}$. In the context of ascending infection, fetal placental inflammation of the umbilical cord has been associated with greater neonatal morbidity and mortality, particularly among preterm pregnancies, than maternal placental inflammation ${ }^{47-49}$. Our study, unlike many others, considers both suboptimal maternal BMI and gestational age, and suggests that increased maternal BMI associates with fetal placental inflammation at preterm, which has been linked to adverse pregnancy and offspring outcomes $^{50,51}$, and maternal placental inflammation at term. Fetal inflammation of the placenta at preterm could thus be an important risk factor for adverse offspring outcomes, and maternal placental inflammation, though less frequently associated with clinical correlates ${ }^{50}$, nonetheless suggests that suboptimal maternal BMI alters gestational tissue histomorphology.

Interestingly, preterm female placentae had increased fetal inflammation, while term male placentae had increased fetal and maternal inflammation. Male placentae have been observed to have higher rates of chronic inflammatory lesions in extreme preterm pregnancies ${ }^{52}$, chronic deciduitis among extreme preterm pregnancies with pre-eclampsia and intrauterine growth restriction ${ }^{53}$, and enrichment in inflammatory pathways ${ }^{54}$. Fewer studies have characterized increased female placental inflammation compared to males, however, female placentae have been shown to have increased chronic villitis in pregnancies complicated by maternal obesity, though this study was restricted to pregnancies at or near term ${ }^{15}$. Our findings suggest that male placentae are more susceptible to inflammation in term pregnancies, which could increase risk for impaired growth or neurodevelopment and long term health outcomes $^{55,56}$, while increased fetal inflammation among preterm female placentae warrants further investigation. Given the associations of maternal obesity and male sex with increased placental inflammation, term males born to mothers with obesity may be most susceptible to placental inflammation. To further investigate this, we conducted an exploratory analysis to assess whether fetal sex associated with placental inflammation among pregnancies with obesity alone. Increased inflammation in male placentae at term only, though not to the same extent as BMI inclusive differences, suggests that both maternal obesity and male sex are independent risk factors for placental inflammation at term.

We found MVM also associated with increased maternal BMI among term pregnancies, consistent with existing data ${ }^{57}$. Previous studies have documented increased maternal vascular lesions ${ }^{44}$ and decidual vasculopathy, but no other lesions associated with MVM, in placentae from term pregnancies complicated by obesity compared to normal weight, although this was attributed to maternal hypertensive disease ${ }^{15}$. MVM lesions impair intervillous blood flow, altering oxygen and nutrient delivery to the fetus ${ }^{32}$, and are associated with adverse offspring outcomes, including preterm birth, intrauterine growth restriction, and small for gestational age infants ${ }^{32}$. Our findings suggest that the adverse environment established by maternal obesity may impair the development and function of placental vasculature, and thus fetal development. Despite known associations of MVM and preterm birth ${ }^{32}$, ${ }^{58}$, increased BMI associated with MVM only among term pregnancies in our cohort ${ }^{15}, 32,44$, suggesting the importance of vascular pathologies across gestation in pregnancies complicated by maternal obesity. Further, male placentae had increased MVM lesions compared to females at term in our study, consistent with other findings of increased decidual vasculopathy in males ${ }^{15}$. Our findings suggest that the placental vasculature pathology may not be so severe as to result in early pregnancy or other adverse perinatal events, but may still have long term implications for the infant.

In contrast to previous studies, we found increased maternal BMI associated with greater odds of having an appropriately mature placenta at term. Placentae from women with obesity have been shown to have immaturity of the villous tree compared to women of normal weight ${ }^{43}$, suggesting structural and functional maladaptation of the vasculature or decreased efficiency in maternal-fetal exchange ${ }^{43}$. We did however observe other placental vascular pathologies, evidenced by increased MVM with higher maternal BMI. The lower number of cases of obesity in our cohort relative to the current rates, and changes to criteria used to 
diagnose distal villous immaturity, may in part account for these differences in apparent placental maturity.

Our findings of decreased placental size and increased infant birthweight to placental weight ratio with lower maternal BMI are consistent with reduced nutrient availability in underweight pregnancies ${ }^{5,20,59}$, and suggest that the placenta may adapt to increase nutrient delivery to the fetus, whereas placentae from higher BMI pregnancies may adapt by regulating nutrient transfer to the fetus in the face of sufficient or overabundance of nutrients ${ }^{60,61}$. Conversely, among term pregnancies, decreased birthweight to placental smallest diameter ratio with lower maternal BMI could suggest lower placental efficiency in pregnancies with underweight. However, placental diameter is reflective of the lateral growth of the placenta and area of the uterine lining that it encompasses, and may be influenced by inter-individual variations such as differences in placental shape, whereas placental weight captures multiple dimensions of placental growth ${ }^{62}$. Further, males had higher birthweight to placental size ratios in both preterm and term pregnancies, consistent with previous studies ${ }^{63}$, suggesting greater placental efficiency than females. Male fetuses tend to grow more rapidly than females and invest greater resources in growth than placental development ${ }^{64}$, and as a result, may be more susceptible to placental insults as well as adverse later health outcomes ${ }^{64}$. Taken together, these results may suggest that males born to mothers who are underweight have the greatest placental efficiency, particularly at term.

Strengths of our study included the large population-based cohort, where previous studies have been limited to animal models of maternal malnutrition or inflammation to assess placental histopathology, or have lacked population size and comprehensive data. Importantly, the large sample size, collection of socioeconomic and demographic factors, deep phenotyping, and prospective nature of the dataset enables a thorough investigation of the relationships between maternal BMI and placental pathologies in a diverse population. Yet, due to the cohort's historical nature, a limitation of our study is that the prevalence of obesity was lower than current rates; only $2.63 \%$ of our cohort were classified as having obesity prepregnancy, compared to $29 \%$ of American women currently ${ }^{65}$. Rates of smoking were also increased during the time of the study compared to rates today (42-45\% during the time of CPP data collection ${ }^{66}$ compared to $16 \%$ in $2019^{67}$ ), and no data were collected on maternal alcohol consumption ${ }^{68}$. Additionally, no data were provided for GDM specifically, which is associated with adverse pregnancy outcomes and is comorbid with obesity ${ }^{69,70}$, however, rates of GDM were much lower during the time of the study than current rates $\left(0.3 \%\right.$ in $1979^{71}$ compared to $7.6 \%$ from $2007-2014^{72}$ ), so any confounding is likely to be minimal. Still, given the population-based design, our findings may be applicable to a broad and diverse population because of the large sample size and thorough collection of demographic and placental pathology data.

Our data demonstrate that compared to normal weight, maternal underweight and obesity prepregnancy, even in the absence of other significant pregnancy complications, are not inert conditions for the developing placenta ${ }^{16,73}$, which may have consequences not only for immediate pregnancy and fetal outcomes but postnatal growth and health trajectories $28,29,74,75$. Characterising placental (mal)adaptations to common maternal conditions using clinically-relevant indicators can help understand the mechanisms through which these conditions affect the developing offspring, and aid clinical decision making to better support high-risk pregnancies and inform interventions to optimise pregnancy, placental, and infant health.

\section{References}

1. Di Angelantonio E, Bhupathiraju SN, Wormser D, et al. Body-mass index and all-cause mortality: individual-participant-data metaanalysis of 239 prospective studies in four continents. The Lancet 2016;388(10046):776-786. doi:10.1016/S0140-6736(16)30175-1

2. Black RE, Victora CG, Walker SP, et al. Maternal and child undernutrition and overweight in low-income and middle-income countries. Lancet. Aug 3 2013;382(9890):427-451. doi:10.1016/s0140-6736(13)60937-x

3. Gotay CC, Katzmarzyk PT, Janssen I, Dawson MY, Aminoltejari K Bartley NL. Updating the Canadian obesity maps: an epidemic in progress. Can J Public Health. Nov 8 2012;104(1):e64-8. doi:10.1007/bf03405657

4. Gaccioli F, Lager S, Powell TL, Jansson T. Placental transport in response to altered maternal nutrition. J Dev Orig Health Dis. Apr 2013;4(2):101-15. doi:10.1017/s2040174412000529

5. Yu Z, Han S, Zhu J, Sun X, Ji C, Guo X. Pre-Pregnancy Body Mass Index in Relation to Infant Birth Weight and Offspring Overweight/Obesity: A Systematic Review and Meta-Analysis. PLOS ONE. 2013;8(4):e61627. doi:10.1371/journal.pone.0061627

6. Basatemur E, Gardiner J, Williams C, Melhuish E, Barnes J, Sutcliffe A. Maternal prepregnancy BMI and child cognition: a longitudinal cohort study. Pediatrics. Jan 2013;131(1):56-63. doi:10.1542/peds.2012-0788

7. Cox B, Tsamou M, Vrijens K, et al. A Co-expression Analysis of the Placental Transcriptome in Association With Maternal Pre-pregnancy BMI and Newborn Birth Weight. Front Genet. 2019;10:354. doi:10.3389/fgene.2019.00354

8. Cotechini T, Hopman WJ, Graham CH. Inflammation-induced fetal growth restriction in rats is associated with altered placental morphometrics. Placenta. 2014/08/01/ 2014;35(8):575-581. doi:https://doi.org/10.1016/j.placenta.2014.05.002

9. Cotechini T, Graham $\mathrm{CH}$. Aberrant maternal inflammation as a cause of pregnancy complications: A potential therapeutic target? Placenta. 2015/08/01/ doi:https://doi.org/10.1016/j.placenta.2015.05.016 2015;36(8):960-966

10. Renaud SJ, Cotechini T, Quirt JS, Macdonald-Goodfellow SK, Othman M, Graham CH. Spontaneous Pregnancy Loss Mediated by Abnormal Maternal Inflammation in Rats Is Linked to Deficient Uteroplacental Perfusion. The Journal of Immunology. 2011;186(3):1799. doi:10.4049/jimmunol.1002679

11. Falcón BJ, Cotechini T, Macdonald-Goodfellow SK, Othman M, Graham $\mathrm{CH}$. Abnormal inflammation leads to maternal coagulopathies associated with placental haemostatic alterations in a rat model of foetal loss. Thromb Haemost. Mar 2012;107(3):438-47. doi:10.1160/th11-09-0626

12. Altmäe $S$, Segura MT, Esteban FJ, et al. Maternal Pre-Pregnancy Obesity Is Associated with Altered Placental Transcriptome. PLoS One. 2017;12(1):e0169223. doi:10.1371/journal.pone.0169223

13. Connor KL, Kibschull M, Matysiak-Zablocki E, et al. Maternal malnutrition impacts placental morphology and transporter expression: an origin for poor offspring growth. The Journal of Nutritional Biochemistry. 2020/04/01/ 2020;78:108329. doi:https://doi.org/10.1016/i.jnutbio.2019.108329

14. Brett KE, Ferraro ZM, Yockell-Lelievre J, Gruslin A, Adamo KB. Maternal-fetal nutrient transport in pregnancy pathologies: the role of the placenta. International journal of molecular sciences. 2014;15(9):16153-16185. doi:10.3390/ijms150916153

15. Leon-Garcia SM, Roeder HA, Nelson KK, et al. Maternal obesity and sex-specific differences in placental pathology. Placenta. 2016/02/01/ 2016;38:33-40. doi:https://doi.org/10.1016/j.placenta.2015.12.006

16. Brouwers L, Franx A, Vogelvang TE, Houben ML, van Rijn BB, Nikkels PG. Association of Maternal Prepregnancy Body Mass Index With Placental Histopathological Characteristics in Uncomplicated 
medRxiv preprint doi: https://doi.org/10.1101/2021.06.01.21258127; this version posted June 4, 2021. The copyright holder for this preprint

(which was not certified by peer review) is the author/funder, who has granted medRxiv a license to display the preprint in perpetuity. All rights reserved. No reuse allowed without permission.

Term Pregnancies. Pediatr Dev Pathol. Jan-Feb 2019;22(1):45-52. doi:10.1177/1093526618785838

17. Bar J, Schreiber L, Saruhanov E, Ben-Haroush A, Golan A, Kovo M. Placental histopathological findings in obese and nonobese women with complicated and uncomplicated pregnancies. Arch Gynecol Obstet. Dec 2012;286(6):1343-7. doi:10.1007/s00404-012-2450-z

18. Khong TY, Mooney EE, Ariel I, et al. Sampling and Definitions of Placental Lesions: Amsterdam Placental Workshop Group Consensus Statement. Archives of Pathology \& Laboratory Medicine. 2016;140(7):698-713. doi:10.5858/arpa.2015-0225-CC

19. Schulz LC, Schlitt JM, Caesar G, Pennington KA. Leptin and the Placental Response to Maternal Food Restriction During Early Pregnancy in Mice1. Biology of Reproduction. 2012;87(5)doi:10.1095/biolreprod.112.103218

20. Belkacemi L, Nelson DM, Desai M, Ross MG. Maternal Undernutrition Influences Placental-Fetal Development1. Biology of Reproduction. 2010;83(3):325-331. doi:10.1095/biolreprod.110.084517

21. Dimasuay KG, Boeuf $P$, Powell TL, Jansson T. Placental Responses to Changes in the Maternal Environment Determine Fetal Growth. 10.3389/fphys.2016.00012. Frontiers in Physiology. 2016;7:12.

22. Myatt L, Maloyan A. Obesity and Placental Function. Semin Reprod Med. //

27.01.2016 2016;34(01):042-049. doi:10.1055/s-0035-1570027

23. Díaz $\mathrm{P}$, Powell TL, Jansson $\mathrm{T}$. The role of placental nutrient sensing in maternal-fetal resource allocation. Biology of reproduction. 2014;91(4):82-82. doi:10.1095/biolreprod.114.121798

24. Catalog NA. Collaborative Perinatal Project Files. 2020. https://catalog.archives.gov/id/606622

25. Seaton SE, King S, Manktelow BN, Draper ES, Field DJ. Babies born at the threshold of viability: changes in survival and workload over 20 years. Archives of disease in childhood Fetal and neonatal edition. 2013;98(1):F15-F20. doi:10.1136/fetalneonatal-2011-301572

26. Gal H, Lysenko $M$, Stroganov $S$, et al. Molecular pathways of senescence regulate placental structure and function. The $E M B O$ journal. doi:10.15252/embj.2018100849

27. Galal M, Symonds I, Murray H, Petraglia F, Smith R. Postterm pregnancy. Facts, views \& vision in ObGyn. 2012;4(3):175-187.

28. Barker DJ, Thornburg KL, Osmond C, Kajantie E, Eriksson JG. The surface area of the placenta and hypertension in the offspring in later life. Int J Dev Biol. 2010;54(2-3):525-30. doi:10.1387/ijdb.082760db

29. Baptiste-Roberts K, Salafia CM, Nicholson WK, Duggan A, Wang NY, Brancati FL. Gross placental measures and childhood growth. J Matern Fetal Neonatal Med. Jan 2009;22(1):13-23. doi:10.1080/14767050802415728

30. Xu Y, Ren L, Zhai S, et al. Association Between Isolated Single Umbilical Artery and Perinatal Outcomes: A Meta-Analysis. Med Sci Monit. Apr 30 2016;22:1451-9. doi:10.12659/msm.897324

31. Redline RW, Ravishankar S. Fetal vascular malperfusion, an update. APMIS. 2018/07/01 2018;126(7):561-569. doi:10.1111/apm.12849

32. Ernst LM. Maternal vascular malperfusion of the placental bed. APMIS. 2018/07/01 2018;126(7):551-560. doi:10.1111/apm.12833

33. Nakayama M. Significance of pathological examination of the placenta, with a focus on intrauterine infection and fetal growth restriction. Journal of Obstetrics and Gynaecology Research. 2017/10/01 2017;43(10):1522-1535. doi:10.1111/jog.13430

34. Wallingford MC, Benson C, Chavkin NW, Chin MT, Frasch MG. Placental Vascular Calcification and Cardiovascular Health: It Is Time to Determine How Much of Maternal and Offspring Health Is Written in Stone. Front Physiol. 2018;9:1044. doi:10.3389/fphys.2018.01044

35. Loukeris K, Sela R, Baergen RN. Syncytial knots as a reflection of placental maturity: reference values for 20 to 40 weeks' gestational age. Pediatr Dev Pathol. Jul-Aug 2010;13(4):305-9. doi:10.2350/0908-0692-0a.1

36. Christians J, Grynspan D. Placental villous hypermaturation is associated with improved neonatal outcomes. Placenta. 01/15 2019;76doi:10.1016/j.placenta.2019.01.012

37. Tang Z, Abrahams V, Mor G, Guller S. Placental Hofbauer cells and complications of pregnancy. Annals of the New York Academy of Sciences. $\quad 03 / 01 \quad 2011 ; 1221: 103-8$. doi:10.1111/j.17496632.2010.05932.x

38. Myrianthopoulos NC, French KS. An application of the U.S. Bureau of the Census socioeconomic index to a large, diversified patient population. Soc Sci Med. Sep 1968;2(3):283-99. doi:10.1016/00377856(68)90004-8

39. Institute of Medicine and National Research Council Committee to Reexamine IOMPWG. The National Academies Collection: Reports funded by National Institutes of Health. In: Rasmussen KM, Yaktine $\mathrm{AL}$, eds. Weight Gain During Pregnancy: Reexamining the Guidelines. National Academies Press (US)

Copyright @ 2009, National Academy of Sciences.; 2009.

40. Stanek J. Comparison of placental pathology in preterm, late-preterm, near-term, and term births. American Journal of Obstetrics \& Gynecology. doi:10.1016/j.ajog.2013.10.015

41. Roberts KA, Riley SC, Reynolds RM, et al. Placental structure and inflammation in pregnancies associated with obesity. Placenta. 2011/03/01/ doi:https://doi.org/10.1016/i.placenta.2010.12.023

42. He M, Curran P, Raker C, Martin S, Larson L, Bourjeily G. Placental findings associated with maternal obesity at early pregnancy. Pathology - Research and Practice. 2016/04/01/ 2016;212(4):282287. doi:https://doi.org/10.1016/j.prp.2016.01.006

43. Loardi C, Falchetti M, Prefumo F, Facchetti F, Frusca T. Placental morphology in pregnancies associated with pregravid obesity. $J$ Matern Fetal Neonatal Med. 2016;29(16):2611-6. doi:10.3109/14767058.2015.1094792

44. Kovo M, Zion-Saukhanov E, Schreiber L, et al. The Effect of Maternal Obesity on Pregnancy Outcome in Correlation With Placental Pathology. Reprod Sci. Dec 2015;22(12):1643-8. doi:10.1177/1933719115592712

45. Hadley EE, Discacciati A, Costantine MM, et al. Maternal obesity is associated with chorioamnionitis and earlier indicated preterm delivery among expectantly managed women with preterm premature rupture of membranes. The Journal of Maternal-Fetal \& Neonatal Medicine. 2019/01/17 doi:10.1080/14767058.2017.1378329

46. Gupta M, Mestan KK, Martin CR, et al. Impact of clinical and histologic correlates of maternal and fetal inflammatory response on gestational age in preterm births. The Journal of Maternal-Fetal \& Neonatal Medicine. 2007/01/01 2007;20(1):39-46. doi:10.1080/14767050601156861

47. Katzman PJ, Metlay LA. Fetal Inflammatory Response is Often Present at Early Stages of Intra-amniotic Infection, and its Distribution along Cord is Variable. Pediatric and Developmental Pathology. 2010/07/01 2010;13(4):265-272. doi:10.2350/09-02-0604-OA.1

48. Kim CJ, Yoon BH, Romero R, et al. Umbilical arteritis and phlebitis mark different stages of the fetal inflammatory response. American Journal of Obstetrics and Gynecology. 2001/08/01/ 2001;185(2):496500. doi:https://doi.org/10.1067/mob.2001.116689

49. Rogers BB, Alexander JM, Head J, Mclntire D, Leveno KJ. Umbilical vein interleukin-6 levels correlate with the severity of placental inflammation and gestational age. Human Pathology. 2002/03/01/ 2002;33(3):335-340. doi:https://doi.org/10.1053/hupa.2002.32214

50. Lau J, Magee F, Qiu Z, Houbé J, Von Dadelszen P, Lee SK. Chorioamnionitis with a fetal inflammatory response is associated with higher neonatal mortality, morbidity, and resource use than 
medRxiv preprint doi: https://doi.org/10.1101/2021.06.01.21258127; this version posted June 4, 2021. The copyright holder for this preprint (which was not certified by peer review) is the author/funder, who has granted medRxiv a license to display the preprint in perpetuity. All rights reserved. No reuse allowed without permission.

chorioamnionitis displaying a maternal inflammatory response only. American Journal of Obstetrics and Gynecology. 2005/09/01/ 2005;193(3):708-713. doi:https://doi.org/10.1016/j.ajog.2005.01.017

51. Tang $Q$, Zhang L, Li H, Shao $Y$. The fetal inflammation response syndrome and adverse neonatal outcomes: a meta-analysis. The Journal of Maternal-Fetal \& Neonatal Medicine. 2019:1-13. doi:10.1080/14767058.2019.1702942

52. Ghidini A, Salafia CM. Gender differences of placental dysfunction in severe prematurity. BJOG: An International Journal of Obstetrics \& Gynaecology. 2005;112(2):140-144. doi:10.1111/j.14710528.2004.00308.x

53. Walker MG, Fitzgerald B, Keating S, Ray JG, Windrim R, Kingdom JCP. Sex-specific basis of severe placental dysfunction leading to extreme preterm delivery. Placenta. 2012/07/01/ 2012;33(7):568-571. doi:https://doi.org/10.1016/i.placenta.2012.03.011

54. Cvitic $\mathrm{S}$, Longtine MS, Hackl $\mathrm{H}$, et al. The human placental sexome differs between trophoblast epithelium and villous vessel endothelium. PloS one. 2013;8(10):e79233-e79233. doi:10.1371/journal.pone.0079233

55. Al-Haddad BJS, Oler E, Armistead B, et al. The fetal origins of mental illness. American Journal of Obstetrics and Gynecology. 2019/12/01/ 2019;221(6):549-562. doi:https://doi.org/10.1016/j.ajog.2019.06.013

56. Romero R, Espinoza J, Gonçalves LF, Kusanovic JP, Friel LA, Nien JK. Inflammation in preterm and term labour and delivery. Semin Fetal Neonatal Med. Oct 2006;11(5):317-26. doi:10.1016/j.siny.2006.05.001

57. Huang L, Liu J, Feng L, Chen Y, Zhang J, Wang W. Maternal prepregnancy obesity is associated with higher risk of placental pathological lesions. Placenta. 2014/08/01/ 2014;35(8):563-569. doi:https://doi.org/10.1016/i.placenta.2014.05.006

58. Scifres CM, Parks WT, Feghali M, Caritis SN, Catov JM. Placental maternal vascular malperfusion and adverse pregnancy outcomes in gestational diabetes mellitus. Placenta. 2017/01/01/ 2017;49:10-15. doi:https://doi.org/10.1016/i.placenta.2016.11.004

59. Liu L, Ma Y, Wang N, Lin W, Liu Y, Wen D. Maternal body mass index and risk of neonatal adverse outcomes in China: a systematic review and meta-analysis. BMC Pregnancy and Childbirth. 2019/03/29 2019;19(1):105. doi:10.1186/s12884-019-2249-z

60. Nogues P, Dos Santos E, Couturier-Tarrade A, et al. Maternal Obesity Influences Placental Nutrient Transport, Inflammatory Status, and Morphology in Human Term Placenta. The Journal of Clinical Endocrinology \& Metabolism. 2020;doi:10.1210/clinem/dgaa660

61. Wallace JM, Horgan GW, Bhattacharya S. Placental weight and efficiency in relation to maternal body mass index and the risk of pregnancy complications in women delivering singleton babies. Placenta. 2012/08/01/ 2012;33(8):611-618. doi:https://doi.org/10.1016/j.placenta.2012.05.006

62. Salafia CM, Maas E, Thorp JM, Eucker B, Pezzullo JC, Savitz DA. Measures of Placental Growth in Relation to Birth Weight and Gestational Age. American Journal of Epidemiology. 2005;162(10):991-998. doi:10.1093/aje/kwi305

63. Wallace JM, Bhattacharya S, Horgan GW. Gestational age, gender and parity specific centile charts for placental weight for singleton deliveries in Aberdeen, UK. Placenta. 2013/03/01/ 2013;34(3):269274. doi:https://doi.org/10.1016/i.placenta.2012.12.007

64. Eriksson JG, Kajantie E, Osmond C, Thornburg K, Barker DJP. Boys live dangerously in the womb. American journal of human biology: the official journal of the Human Biology Council. May-Jun 2010;22(3):330-335. doi:10.1002/ajhb.20995

65. Driscoll A, Gregory E. Increases in Prepregnancy Obesity: United States, 2016-2019. https://www.cdc.gov/nchs/products/databriefs/db392.htm

66. (US) OoSaH. Chapter 2. Patterns of Tobacco Use Among Women and Girls. Text. 2001/03 2001;doi:https://www.ncbi.nlm.nih.gov/books/NBK44311/
67. Cornelius M, Wang T, Jamal A, Loretan C, Neff L. Tobacco Product Use Among Adults - United States, 2019 https://www.cdc.gov/mmwr/volumes/69/wr/mm6946a4.htm?s cid=m m6946a4 w

68. Klebanoff MA. The Collaborative Perinatal Project: a 50-year retrospective. Paediatric and perinatal epidemiology. 2009;23(1):2-8. doi:10.1111/j.1365-3016.2008.00984.x

69. Al-Goblan AS, Al-Alfi MA, Khan MZ. Mechanism linking diabetes mellitus and obesity. Diabetes, metabolic syndrome and obesity : targets and therapy. 2014;7:587-591. doi:10.2147/DMSO.S67400

70. Huynh J, Dawson D, Roberts D, Bentley-Lewis R. A systematic review of placental pathology in maternal diabetes mellitus. Placenta. 2015;36(2):101-114. doi:10.1016/j.placenta.2014.11.021

71. Lavery JA, Friedman AM, Keyes KM, Wright JD, Ananth CV. Gestational diabetes in the United States: temporal changes in prevalence rates between 1979 and 2010. https://doi.org/10.1111/1471-0528.14236. BJOG: An International Journal of Obstetrics \& Gynaecology. 2017/04/01 2017;124(5):804813. doi:https://doi.org/10.1111/1471-0528.14236

72. Casagrande SS, Linder B, Cowie CC. Prevalence of gestational diabetes and subsequent Type 2 diabetes among U.S. women. Diabetes Res Clin Pract. Jul 2018;141:200-208. doi:10.1016/j.diabres.2018.05.010

73. Romero R, Kim YM, Pacora P, et al. The frequency and type of placental histologic lesions in term pregnancies with normal outcome. Journal of perinatal medicine. 2018;46(6):613-630. doi:10.1515/.jpm2018-0055

74. Marciniak A, Patro-Małysza J, Kimber-Trojnar Ż, Marciniak B, Oleszczuk J, Leszczyńska-Gorzelak B. Fetal programming of the metabolic syndrome. Taiwanese Journal of Obstetrics and Gynecology. 2017/04/01/ 2017;56(2):133-138. doi:https://doi.org/10.1016/j.tjog.2017.01.001

75. Godfrey KM. The Role of the Placenta in Fetal Programming-A Review. Placenta. 2002/04/01/ 2002;23:S20-S27. doi:https://doi.org/10.1053/plac.2002.0773 
medRxiv preprint doi: https://doi.org/10.1101/2021.06.01.21258127; this version posted June 4, 2021. The copyright holder for this preprint (which was not certified by peer review) is the author/funder, who has granted medRxiv a license to display the preprint in perpetuity.

All rights reserved. No reuse allowed without permission.

\section{Supplementary tables}

Supplementary Table 1. Binary categories for placental pathology variables for multivariable analysis.

\begin{tabular}{ll}
\hline Categorical variable & Collapsed binary categories \\
\hline Cord number of vessels & Three vessels vs two vessels \\
\hline $\begin{array}{l}\text { Cord edema } \\
\text { (derived from “cord description" variable) }\end{array}$ & Presence of cord edema vs absence of cord edema \\
\hline Opacity of membranes & Not opaque vs any degree of opacity (partially opaque, opaque) \\
\hline Thrombosed fetal vessel & Not seen vs present \\
\hline $\begin{array}{l}\text { Placental infarcts } \\
\text { (derived from "infarcts size" variable) }\end{array}$ & $\begin{array}{l}\text { No infarcts seen (not applicable) vs any infarcts seen (all infarcts less than three centimetres, at least one infarct measures } \\
\text { three or more centimetres) }\end{array}$ \\
\hline Cut surface calcification & Not seen (none) vs any calcification seen (maternal surface only, calcification throughout) \\
\hline Neutrophilic infiltration & Not seen vs any degree of neutrophilic infiltration seen (slight, moderate, or marked) \\
\hline Decidual vessels fibrinoid & Not seen vs present \\
\hline Decidual vessels atheroma & Not seen vs present \\
\hline Langhan's layer & Not seen vs present \\
\hline Syncytium nuclear clumping & Normal/less than normal for term placenta vs excessive for term placenta/excessive in parts \\
\hline Hofbauer cells & Few vs many \\
\hline Stromal fibrosis & Not seen vs present (present- not qualified further, present- rare, present- many) \\
\hline Pathological edema & Not seen vs present (present- not qualified further, present- rare, present- many) \\
\hline Apparent maturity of the placenta & $\begin{array}{l}\text { Preterm pregnancies: appropriate mature }(\leq 36 \text { weeks) vs hyper-mature }(\geq 37 \text { weeks) } \\
\text { Term pregnancies: appropriate mature }(\geq 37 \text { weeks) vs immature }(\leq 36 \text { weeks) }\end{array}$ \\
\hline
\end{tabular}


medRxiv preprint doi: https://doi.org/10.1101/2021.06.01.21258127; this version posted June 4, 2021. The copyright holder for this preprint (which was not certified by peer review) is the author/funder, who has granted medRxiv a license to display the preprint in perpetuity.

All rights reserved. No reuse allowed without permission.

Supplementary Table 2. Derived summary scores variable coding.

\begin{tabular}{l}
\hline Summary score \\
\hline Maternal inflammation summary score \\
\hline Fetal inflammation summary score \\
Maternal vascular malperfusion (MVM) summary score
\end{tabular}

Immaturity summary score

Variable coding

Opacity of membranes: non-opaque membranes coded as zero, partially opaque as one, and opaque as two Neutrophilic infiltration of the amnion membrane, chorion membrane, amnion of the placenta, and chorion of the placenta: no neutrophilic infiltration coded as zero, slight infiltration coded as one, moderate coded as two, and marked coded as three

Neutrophilic infiltration of the umbilical vein, artery, and cord substance: no neutrophilic infiltration coded as zero, slight infiltration coded as one, moderate coded as two, and marked coded as three

Presence of infarcts: any infarcts seen coded as one Syncytium-nuclear clumping: excessive syncytium-nuclear clumping coded as one

Langhans' layer: presence of Langhans' layer coded as one

Hofbauer cells: many Hofbauer cells coded as one

Stromal fibrosis: no stromal fibrosis coded as one

Syncytium-nuclear clumping: normal or less than normal syncytium-nuclear clumping coded as one 
medRxiv preprint doi: https://doi.org/10.1101/2021.06.01.21258127; this version posted June 4, 2021. The copyright holder for this preprint (which was not certified by peer review) is the author/funder, who has granted medRxiv a license to display the preprint in perpetuity. All rights reserved. No reuse allowed without permission.

Supplementary Table 3. Maternal characteristics by prepregnancy BMI in preterm pregnancies, N=1739.

\begin{tabular}{|c|c|c|c|c|c|c|}
\hline \multicolumn{7}{|c|}{ Prepregnancy BMI } \\
\hline & $\begin{array}{l}\text { UW } \\
(\mathrm{n}=259)\end{array}$ & $\begin{array}{l}\text { NW } \\
(n=1286)\end{array}$ & $\begin{array}{l}\text { OW } \\
(n=156)\end{array}$ & $\begin{array}{l}\mathrm{OB} \\
(\mathrm{n}=38)\end{array}$ & p-value & Effect size \\
\hline \multicolumn{7}{|l|}{ Maternal characteristics } \\
\hline Prepregnancy BMI & $17.7(17.0,18.2)^{\mathrm{A}}$ & $20.9(19.8,22.3)^{\mathrm{B}}$ & $26.3(25.5,27.5)^{\mathrm{C}}$ & $31.9(30.2,34.9)^{\mathrm{D}}$ & $<0.0001$ & $0.65(0.62-0.67)$ \\
\hline Gestational weight gain max (lbs) & $23(16,28)^{\mathrm{A}}$ & $19(13,26)^{\mathrm{B}}$ & $19(11,28.5)^{\mathrm{AB}}$ & $17(7.5,30)^{\mathrm{AB}}$ & 0.005 & $-0.09(-0.14$ to -0.04$)$ \\
\hline 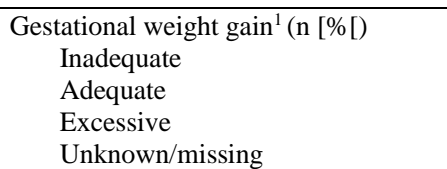 & $\begin{array}{l}180(69.5) \\
67(25.9) \\
7(2.70) \\
5(1.93)\end{array}$ & $\begin{array}{l}866(67.3) \\
305(23.7) \\
115(8.94) \\
0\end{array}$ & $\begin{array}{l}50(32.1) \\
50(32.1) \\
56(35.9) \\
0\end{array}$ & $\begin{array}{l}10(26.3) \\
9(23.7) \\
19(50.0) \\
0\end{array}$ & $<0.0001$ & $0.24(0.20-0.27)$ \\
\hline $\begin{array}{l}\text { Diabetes mellitus }{ }^{2} \text { (n [\%[) } \\
\text { Yes } \\
\text { No } \\
\text { Other/unknown/missing }\end{array}$ & $\begin{array}{l}4(1.54) \\
253(97.7) \\
2(0.77) \\
\end{array}$ & $\begin{array}{l}24(1.87) \\
1257(97.7) \\
5(0.39) \\
\end{array}$ & $\begin{array}{l}1(0.64) \\
154(98.7) \\
1(0.64)\end{array}$ & $\begin{array}{l}1(2.63) \\
37(97.4) \\
0\end{array}$ & 0.62 & $0.03(0.00-0.06)$ \\
\hline Maternal age (years) & $18(16,20)^{\mathrm{A}}$ & $18(16,20)^{\mathrm{A}}$ & $19(17,22)^{\mathrm{B}}$ & $22.5(19,26)^{\mathrm{C}}$ & $<0.0001$ & $0.04(-0.01$ to 0.09$)$ \\
\hline $\begin{array}{l}\text { Maternal age grouped (years) (n [\%]) } \\
\quad \leq 19 \\
20-24 \\
25-29 \\
\geq 30 \\
\quad \text { Unknown/missing } \\
\end{array}$ & $\begin{array}{l}177(68.3) \\
70(27.0) \\
9(3.47) \\
3(1.16) \\
0\end{array}$ & $\begin{array}{l}880(68.4) \\
329(25.6) \\
57(4.43) \\
20(1.56) \\
0\end{array}$ & $\begin{array}{l}84(53.4) \\
49(31.4) \\
18(11.5) \\
5(3.21) \\
0 \\
\end{array}$ & $\begin{array}{l}15(39.5) \\
12(31.6) \\
4(10.5) \\
7(18.42) \\
0\end{array}$ & $<0.0001$ & $0.13(0.09-0.15)$ \\
\hline $\begin{array}{l}\text { Maternal race (n [\%]) } \\
\text { Black } \\
\text { White } \\
\text { Other } \\
\text { Unknown/missing } \\
\end{array}$ & $\begin{array}{l}168(64.9) \\
71(27.4) \\
20(7.72) \\
0\end{array}$ & $\begin{array}{l}828(64.4) \\
339(26.4) \\
119(9.25) \\
0\end{array}$ & $\begin{array}{l}112(71.8) \\
32(20.5) \\
12(7.69) \\
0\end{array}$ & $\begin{array}{l}31(81.6) \\
5(13.2) \\
2(5.26) \\
0\end{array}$ & 0.16 & $0.05(0.00-0.07)$ \\
\hline $\begin{array}{l}\text { Maternal education (years) (n [\%]) } \\
\text { Less than high school }(\leq 9) \\
\text { High school }(10-12) \\
\text { College and above }(>12) \\
\text { Unknown/missing }\end{array}$ & $\begin{array}{l}103(39.8) \\
137(52.9) \\
14(5.41) \\
5(1.93) \\
\end{array}$ & $\begin{array}{l}419(32.6) \\
729(56.7) \\
116(9.02) \\
22(1.71) \\
\end{array}$ & $\begin{array}{l}45(28.9) \\
96(61.5) \\
13(8.33) \\
2(1.28)\end{array}$ & $\begin{array}{l}13(34.2) \\
22(57.9) \\
2(5.26) \\
1(2.63)\end{array}$ & 0.13 & $0.05(0.00-0.07)$ \\
\hline $\begin{array}{l}\text { Marital status (n [\%]) } \\
\text { Single } \\
\text { Married/common law } \\
\text { Widowed/divorced/separated } \\
\text { Unknown/missing } \\
\end{array}$ & $\begin{array}{l}120(46.3) \\
136(52.5) \\
3(1.16) \\
0\end{array}$ & $\begin{array}{l}602(46.8) \\
668(51.9) \\
16(1.24) \\
0 \\
\end{array}$ & $\begin{array}{l}77(49.4) \\
74(47.4) \\
5(3.21) \\
0\end{array}$ & $\begin{array}{l}19(50.0) \\
19(50.0) \\
0 \\
0\end{array}$ & 0.55 & $0.04(0.00-0.06)$ \\
\hline $\begin{array}{l}\text { Family income }^{3}(\mathrm{n}[\%]) \\
\leq \$ 1999 \\
\$ 2000-4999 \\
\$ 5000-7999 \\
\geq \$ 8000 \\
\text { Unknown/missing }\end{array}$ & $\begin{array}{l}57(22.0) \\
121(46.7) \\
34(13.1) \\
7(2.70) \\
40(15.4) \\
\end{array}$ & $\begin{array}{l}247(19.2) \\
626(48.7) \\
163(12.7) \\
72(5.60) \\
178(13.8) \\
\end{array}$ & $\begin{array}{l}29(18.6) \\
71(45.5) \\
26(16.7) \\
4(2.56) \\
26(16.7)\end{array}$ & $\begin{array}{l}6(15.8) \\
18(47.4) \\
8(21.1) \\
0 \\
6(15.8) \\
\end{array}$ & 0.10 & $0.05(0.00-0.07)$ \\
\hline Socioeconomic index & $3.6(2.0,5.0)$ & $3.7(2.3,5.3)$ & $3.7(2.0,5.3)$ & $3.85(2.55,5.23)$ & 0.26 & $0.05(0.0002-0.10)$ \\
\hline $\begin{array}{l}\text { Socioeconomic index grouped }(\mathrm{n}[\%]) \\
0.0-1.9 \\
2.0-3.9 \\
4.0-5.9 \\
6.0-7.9 \\
8.0-9.5 \\
\text { Unknown/missing } \\
\end{array}$ & $\begin{array}{l}48(18.5) \\
94(36.3) \\
61(23.6) \\
36(13.9) \\
11(4.25) \\
9(3.47) \\
\end{array}$ & $\begin{array}{l}185(14.4) \\
449(34.9) \\
365(28.4) \\
167(13.0) \\
88(6.84) \\
32(2.49) \\
\end{array}$ & $\begin{array}{l}27(17.3) \\
52(33.3) \\
46(29.5) \\
20(12.8) \\
9(5.77) \\
2(1.28) \\
\end{array}$ & $\begin{array}{l}5(13.2) \\
13(34.2) \\
13(34.2) \\
4(10.5) \\
1(2.63) \\
2(5.26) \\
\end{array}$ & 0.64 & $0.04(0.00-0.05)$ \\
\hline $\begin{array}{l}\text { Housing density }(\mathrm{n}[\%[) \\
\quad<1 \text { person } \\
1.0-3.0 \text { people } \\
>3 \text { people } \\
\text { Other } 4 \text { unknown/missing }\end{array}$ & $\begin{array}{l}68(26.3) \\
177(68.3) \\
8(3.09) \\
6(2.32) \\
\end{array}$ & $\begin{array}{l}322(25.0) \\
897(69.8) \\
40(3.11) \\
27(2.10) \\
\end{array}$ & $\begin{array}{l}43(27.6) \\
109(69.9) \\
3(1.92) \\
1(0.64) \\
\end{array}$ & $\begin{array}{l}12(31.6) \\
25(65.8) \\
0 \\
1(2.63) \\
\end{array}$ & 0.67 & $0.03(0.00-0.04)$ \\
\hline $\begin{array}{l}\text { Smoking history }^{5}(\mathrm{n}[\%[) \\
\text { Non-smokers } \\
\text { Light smokers ( }<1 \text { pack per day) } \\
\text { Heavy smokers ( } \geq 1 \text { pack per day }) \\
\text { Unknown/missing }\end{array}$ & $\begin{array}{l}150(57.9) \\
85(32.8) \\
21(8.11) \\
3(1.16) \\
\end{array}$ & $\begin{array}{l}732(56.9) \\
452(35.2) \\
92(7.15) \\
10(0.78) \\
\end{array}$ & $\begin{array}{l}89(57.1) \\
53(34.0) \\
13(8.33) \\
1(0.64)\end{array}$ & $\begin{array}{l}23(60.5) \\
14(36.8) \\
0 \\
1(2.63)\end{array}$ & 0.37 & $0.03(0.00-0.05)$ \\
\hline \multicolumn{7}{|l|}{ Infant characteristics } \\
\hline $\begin{array}{l}\text { Infant sex (n }[\%]) \\
\text { Male } \\
\text { Female }\end{array}$ & $\begin{array}{l}140(54.1) \\
119(45.9)\end{array}$ & $\begin{array}{l}675(52.5) \\
611(47.5) \\
\end{array}$ & $\begin{array}{l}69(44.2) \\
87(55.8) \\
\end{array}$ & $\begin{array}{l}22(57.9) \\
16(42.1)\end{array}$ & 0.18 & $0.05(0.00-0.09)$ \\
\hline Gestational age (weeks) & $34(32,36)$ & $34(32,36)$ & $34(31,36)$ & $34(29.75,36)$ & 0.57 & $-0.01(-0.06$ to 0.04$)$ \\
\hline
\end{tabular}


medRxiv preprint doi: https://doi.org/10.1101/2021.06.01.21258127; this version posted June 4, 2021. The copyright holder for this preprint (which was not certified by peer review) is the author/funder, who has granted medRxiv a license to display the preprint in perpetuity.

All rights reserved. No reuse allowed without permission.

Data are median (IQR; Kruskal-Wallis/Wilcoxon test for non-parametric data with Steel-Dwass post-hoc) for continuous variables, or n (\%) (Likelihood Ratio Chi Square test) for categorical variables. *p $<0.05$. Effect sizes are Wilcoxon test effect size $(\mathrm{r})(95 \% \mathrm{CI})$ for continuous variables, or $\mathrm{Cramer}$ 's $\mathrm{V}(95 \% \mathrm{CI})$ for categorical variables. ${ }^{1}$ Weight gain recommendations based on Institute of Medicine (2009) guidelines for singleton pregnancies based on prepregnancy BMI. ${ }^{2}$ Includes diabetes mellitus before pregnancy, during pregnancy, before and during pregnancy, during and post pregnancy, or before, during and post pregnancy. Does not include maternal diabetes mellitus during post partum period only. ${ }^{3} \$ 1999, \$ 2000-4999, \$ 5000-7999, \geq \$ 8000$ equivalent to $\$ 17315, \leq \$ 17800, \$ 17801-\$ 44500, \$ 44501-\$ 71200, \geq \$ 71201$ in 2020 US dollars, respectively. ${ }^{4}$ Gravida in home for unwed mothers. ${ }^{5} 1$ pack is 20 cigarettes. UW $=$ underweight. $\mathrm{NW}=$ normal weight. OW $=$ overweight. $\mathrm{OB}=$ obese. 
medRxiv preprint doi: https://doi.org/10.1101/2021.06.01.21258127; this version posted June 4, 2021. The copyright holder for this preprint (which was not certified by peer review) is the author/funder, who has granted medRxiv a license to display the preprint in perpetuity. All rights reserved. No reuse allowed without permission.

Supplementary Table 4. Maternal characteristics by prepregnancy BMI in term pregnancies, N=10,415.

\begin{tabular}{|c|c|c|c|c|c|c|}
\hline \multicolumn{7}{|c|}{ Prepregnancy BMI } \\
\hline & $\begin{array}{l}\text { UW } \\
(n=1201)\end{array}$ & $\begin{array}{l}\text { NW } \\
(n=8010)\end{array}$ & $\begin{array}{l}\text { OW } \\
(\mathrm{n}=922)\end{array}$ & $\begin{array}{l}\text { OB } \\
(\mathrm{n}=282)\end{array}$ & p-value & Effect size \\
\hline \multicolumn{7}{|l|}{ Maternal characteristics } \\
\hline Prepregnancy BMI & $17.8(17.2,18.1)^{\mathrm{A}}$ & $21.0(19.8,22.3)^{\mathrm{B}}$ & $26.5(25.6,27.5)^{\mathrm{C}}$ & $32.0(30.8,34.4)^{\mathrm{D}}$ & $<0.0001$ & $0.58(0.57-0.60)$ \\
\hline Gestational weight gain max (lbs) & $25(20,31)^{\mathrm{A}}$ & $24(18,30)^{\mathrm{B}}$ & $23(15,31)^{\mathrm{B}}$ & $20(13,28.5)^{\mathrm{C}}$ & $<0.0001$ & $-0.05(-0.07$ to -0.03$)$ \\
\hline $\begin{array}{l}\text { Gestational weight } \text { gain }^{1}(\mathrm{n}[\%[) \\
\text { Inadequate } \\
\text { Adequate } \\
\text { Excessive } \\
\text { Unknown/missing }\end{array}$ & $\begin{array}{l}749(62.4) \\
396(33.0) \\
54(4.50) \\
2(0.17)\end{array}$ & $\begin{array}{l}4287(53.5) \\
2798(34.9) \\
864(10.8) \\
61(0.76)\end{array}$ & $\begin{array}{l}208(22.6) \\
320(34.7) \\
365(39.6) \\
29(3.15)\end{array}$ & $\begin{array}{l}45(16.0) \\
82(29.1) \\
122(43.3) \\
33(11.7)\end{array}$ & $<0.0001$ & $0.23(0.21-0.24)$ \\
\hline $\begin{array}{l}\text { Diabetes mellitus }^{2}(\mathrm{n}[\%[) \\
\text { Yes } \\
\text { No } \\
\text { Other/unknown/missing }\end{array}$ & $\begin{array}{l}13(1.08) \\
1181(98.3) \\
7(0.58)\end{array}$ & $\begin{array}{l}47(0.59) \\
7940(99.1) \\
23(0.29)\end{array}$ & $\begin{array}{l}8(0.87) \\
913(99.0) \\
1(0.11)\end{array}$ & $\begin{array}{l}3(1.06) \\
279(98.9) \\
0\end{array}$ & 0.21 & $0.02(0.00-0.04)$ \\
\hline Maternal age (years) & $19(17,21)^{\mathrm{A}}$ & $19(17,22)^{\mathrm{A}}$ & $20(18,22)^{\mathrm{B}}$ & $21(18.75,25)^{\mathrm{C}}$ & $<0.0001$ & $0.05(0.03-0.07)$ \\
\hline $\begin{array}{l}\text { Maternal age grouped (years) (n [\%]) } \\
\quad \leq 19 \\
20-24 \\
25-29 \\
\geq 30 \\
\quad \text { Missing }\end{array}$ & $\begin{array}{l}692(57.6) \\
403(33.6) \\
92(7.66) \\
14(1.17) \\
0\end{array}$ & $\begin{array}{l}4132(51.6) \\
3030(37.8) \\
652(8.14) \\
196(2.45) \\
0\end{array}$ & $\begin{array}{l}435(47.2) \\
355(38.5) \\
88(9.54) \\
44(4.77) \\
0\end{array}$ & $\begin{array}{l}91(32.3) \\
120(42.6) \\
36(12.8) \\
35(12.41) \\
0\end{array}$ & $<0.0001$ & $0.07(0.06-0.08)$ \\
\hline $\begin{array}{l}\text { Maternal race (n [\%]) } \\
\text { Black } \\
\text { White } \\
\text { Other } \\
\text { Missing } \\
\end{array}$ & $\begin{array}{l}528(44.0) \\
546(45.5) \\
127(10.6) \\
0\end{array}$ & $\begin{array}{l}3309(41.3) \\
4138(51.7) \\
563(7.03) \\
0 \\
\end{array}$ & $\begin{array}{l}437(47.4) \\
412(44.7) \\
73(7.92) \\
0\end{array}$ & $\begin{array}{l}162(57.5) \\
108(38.3) \\
12(4.26) \\
0\end{array}$ & $<0.0001$ & $0.06(0.04-0.07)$ \\
\hline $\begin{array}{l}\text { Maternal education (years) (n [\%]) } \\
\text { Less than high school }(\leq 9) \\
\text { High school }(10-12) \\
\text { College and above }(>12) \\
\text { Unknown/missing }\end{array}$ & $\begin{array}{l}327(27.2) \\
663(55.2) \\
202(16.8) \\
9(0.75)\end{array}$ & $\begin{array}{l}1668(20.8) \\
4572(57.1) \\
1682(21.0) \\
88(1.10) \\
\end{array}$ & $\begin{array}{l}227(24.6) \\
564(61.2) \\
111(12.0) \\
20(2.17) \\
\end{array}$ & $\begin{array}{l}84(29.8) \\
172(61.0) \\
24(8.51) \\
2(0.71) \\
\end{array}$ & $<0.0001$ & $0.07(0.05-0.08)$ \\
\hline $\begin{array}{l}\text { Marital status (n [\%]) } \\
\text { Single } \\
\text { Married/common law } \\
\text { Widowed/divorced/separated } \\
\text { Unknown/missing } \\
\end{array}$ & $\begin{array}{l}389(32.4) \\
785(65.4) \\
27(2.25) \\
0\end{array}$ & $\begin{array}{l}2381(29.7) \\
5474(68.3) \\
155(1.94) \\
0 \\
\end{array}$ & $\begin{array}{l}313(34.0) \\
593(64.3) \\
16(1.74) \\
0\end{array}$ & $\begin{array}{l}95(33.7) \\
176(62.4) \\
11(3.90) \\
0\end{array}$ & 0.01 & $0.03(0.008-0.04)$ \\
\hline $\begin{array}{l}\text { Family income }^{3}(\mathrm{n}[\%]) \\
\leq \$ 1999 \\
\$ 2000-4999 \\
\$ 5000-7999 \\
\geq \$ 8000 \\
\quad \text { Unknown/missing }\end{array}$ & $\begin{array}{l}183(15.2) \\
570(47.5) \\
226(18.8) \\
93(7.74) \\
129(10.7)\end{array}$ & $\begin{array}{l}1202(15.0) \\
3572(44.6) \\
1556(19.4) \\
808(10.1) \\
872(10.9)\end{array}$ & $\begin{array}{l}148(16.1) \\
422(45.8) \\
178(19.3) \\
72(7.81) \\
102(11.1)\end{array}$ & $\begin{array}{l}44(15.6) \\
140(49.7) \\
61(21.6) \\
13(4.61) \\
24(8.51)\end{array}$ & 0.005 & $0.03(0.006-0.03)$ \\
\hline Socioeconomic index & $4.3(3.0,6.7)^{\mathrm{A}}$ & $5.0(3.3,7.0)^{\mathrm{B}}$ & $4.4(3.0,6.3)^{\mathrm{A}}$ & $4.3(2.7,5.7)^{\mathrm{A}}$ & $<0.0001$ & $0.05(0.03-0.07)$ \\
\hline $\begin{array}{l}\text { Socioeconomic index grouped }(\mathrm{n}[\%]) \\
0.0-1.9 \\
2.0-3.9 \\
4.0-5.9 \\
6.0-7.9 \\
8.0-9.5 \\
\text { Unknown/missing }\end{array}$ & $\begin{array}{l}126(10.5) \\
351(29.2) \\
318(26.5) \\
223(18.6) \\
165(13.7) \\
18(1.50)\end{array}$ & $\begin{array}{l}671(8.38) \\
1990(24.8) \\
2098(26.2) \\
1776(22.2) \\
1343(16.8) \\
132(1.65)\end{array}$ & $\begin{array}{l}86(9.33) \\
281(30.5) \\
264(28.6) \\
184(20.0) \\
77(8.35) \\
30(3.25)\end{array}$ & $\begin{array}{l}28(9.93) \\
86(30.5) \\
99(35.1) \\
46(16.3) \\
11(3.90) \\
12(4.26)\end{array}$ & $<0.0001$ & $0.06(0.05-0.07)$ \\
\hline $\begin{array}{l}\text { Housing density }(\mathrm{n}[\%[) \\
<1 \text { person } \\
1.0-3.0 \text { people } \\
>3 \text { people } \\
\text { Other }{ }^{4} / \text { unknown } / \text { missing }\end{array}$ & $\begin{array}{l}342(28.5) \\
820(68.3) \\
24(2.00) \\
15(1.25)\end{array}$ & $\begin{array}{l}2679(33.5) \\
5095(63.6) \\
109(1.36) \\
127(1.59)\end{array}$ & $\begin{array}{l}331(35.9) \\
557(60.4) \\
7(0.76) \\
27(2.93)\end{array}$ & $\begin{array}{l}91(32.3) \\
185(65.6) \\
0 \\
6(2.13)\end{array}$ & $<0.0001$ & $0.04(0.02-0.05)$ \\
\hline $\begin{array}{l}\text { Smoking history }^{5} \text { (n }[\%[) \\
\text { Non-smokers } \\
\text { Light smokers }(<1 \text { pack per day) } \\
\text { Heavy smokers }(\geq 1 \text { pack per day) } \\
\text { Unknown/missing }\end{array}$ & $\begin{array}{l}675(56.2) \\
392(32.6) \\
130(10.8) \\
4(0.33)\end{array}$ & $\begin{array}{l}4751(59.3) \\
2511(31.4) \\
719(8.98) \\
29(0.36)\end{array}$ & $\begin{array}{l}537(58.24) \\
281(30.5) \\
100(10.9) \\
4(0.43)\end{array}$ & $\begin{array}{l}162(57.5) \\
82(29.1) \\
34(12.1) \\
4(1.42)\end{array}$ & 0.09 & $0.02(0.00-0.03)$ \\
\hline \multicolumn{7}{|l|}{ Infant characteristics } \\
\hline $\begin{array}{l}\text { Infant sex (n [\%]) } \\
\text { Male } \\
\text { Female }\end{array}$ & $\begin{array}{l}624(52.0) \\
577(48.0)\end{array}$ & $\begin{array}{l}4117(51.4) \\
3893(48.6)\end{array}$ & $\begin{array}{l}481(52.2) \\
441(47.8)\end{array}$ & $\begin{array}{l}135(47.9) \\
147(52.1)\end{array}$ & 0.62 & $0.01(0.00-0.03)$ \\
\hline Gestational age (weeks) & $40(39,41)^{\mathrm{A}}$ & $40(39,41)^{\mathrm{A}}$ & $40(39,41)^{\mathrm{A}}$ & $40(39,41)^{\mathrm{A}}$ & 0.02 & $0.01(-0.00$ to 0.03$)$ \\
\hline
\end{tabular}


medRxiv preprint doi: https://doi.org/10.1101/2021.06.01.21258127; this version posted June 4, 2021. The copyright holder for this preprint (which was not certified by peer review) is the author/funder, who has granted medRxiv a license to display the preprint in perpetuity.

All rights reserved. No reuse allowed without permission.

Data are median (IQR; Kruskal-Wallis/Wilcoxon test for non-parametric data with Steel-Dwass post-hoc) for continuous variables, or n (\%) (Likelihood Ratio Chi Square test) for categorical variables. ${ }^{*} \mathrm{p}<0.05$. Effect sizes are Wilcoxon test effect size (r) for continuous variables, or Cramer's V for categorical variables. ${ }^{1}$ Weight gain recommendations based on Institute of Medicine (2009) guidelines for singleton pregnancies based on prepregnancy BMI. ${ }^{2}$ Includes diabetes mellitus before pregnancy, during pregnancy, before and during pregnancy, during and post pregnancy, or before, during and post pregnancy. Does not include maternal diabetes mellitus during post partum period only. ${ }^{3} \$ 1999, \$ 2000-4999, \$ 5000-7999, \geq \$ 8000$ equivalent to $\$ 17315, \leq \$ 17800, \$ 17801-\$ 44500, \$ 44501-\$ 71200, \geq \$ 71201$ in 2020 US dollars, respectively. ${ }^{4}$ Gravida in home for unwed mothers. ${ }^{5} 1$ pack is 20 cigarettes. UW $=$ underweight. $\mathrm{NW}=$ normal weight. $\mathrm{OW}=$ overweight. $\mathrm{OB}=$ obese. 
Supplementary Table 5. Associations between maternal prepregnancy BMI and macroscopic placental pathologies in term pregnancies, N=10,145.

\begin{tabular}{|c|c|c|c|c|c|c|}
\hline \multirow[t]{2}{*}{ Placental pathology } & \multicolumn{4}{|c|}{ Prepregnancy BMI } & \multirow[b]{2}{*}{ p-value } & \multirow[b]{2}{*}{ Effect size } \\
\hline & $\begin{array}{l}\text { UW } \\
(n=1201)\end{array}$ & $\begin{array}{l}\mathrm{NW} \\
(\mathrm{n}=8010)\end{array}$ & $\begin{array}{l}\text { OW } \\
(n=922)\end{array}$ & $\begin{array}{l}\text { OB } \\
(n=282)\end{array}$ & & \\
\hline \multicolumn{7}{|l|}{ Placenta } \\
\hline Placenta shape & & & & & 0.57 & $0.03(0.00-0.03)$ \\
\hline Non-partite & $1059(88.2)$ & $7230(90.2)$ & 847 (91.9) & $256(90.8)$ & & \\
\hline Bipartite & $14(1.17)$ & $107(1.34)$ & $8(0.87)$ & $4(1.42)$ & & \\
\hline Tripartite & 0 & $1(0.01)$ & $1(0.11)$ & 0 & & \\
\hline Succenturiate & $19(1.58)$ & $83(1.04)$ & $10(1.08)$ & $2(0.71)$ & & \\
\hline Membranous placenta & 0 & $1(0.01)$ & $1(0.11)$ & 0 & & \\
\hline Crescent shaped & $1(0.08)$ & $1(0.01)$ & 0 & 0 & & \\
\hline Irregular shape & $3(0.25)$ & $38(0.47)$ & $5(0.54)$ & 0 & & \\
\hline Unknown/missing & $105(8.74)$ & $549(6.85)$ & $50(5.42)$ & 20 (7.09) & & \\
\hline Weight (g) & $400(352,455)^{\mathrm{A}}$ & $420(370,480)^{\mathrm{B}}$ & $440(380,500)^{\mathrm{C}}$ & $447(380,500)^{\mathrm{BC}}$ & $<0.0001$ & $0.08(0.06-0.10)$ \\
\hline Largest diameter $(\mathrm{cm})$ & $19(17,20)^{\mathrm{A}}$ & $19(18,20)^{\mathrm{B}}$ & $19(18,20)^{\mathrm{C}}$ & $19(18,20)^{\mathrm{BC}}$ & $<0.0001$ & $0.06(0.04-0.08)$ \\
\hline Smallest diameter $(\mathrm{cm})$ & $16(15,17)^{\mathrm{A}}$ & $16(15,18)^{\mathrm{B}}$ & $17(15,18)^{\mathrm{C}}$ & $16.5(15,18)^{\mathrm{BC}}$ & $<0.0001$ & $0.05(0.03-0.07)$ \\
\hline Thickness (mm) & $20(20,25)^{\mathrm{A}}$ & $20(20,25)^{\mathrm{A}}$ & $21(20,25)^{\mathrm{A}}$ & $20(20,25)^{\mathrm{A}}$ & 0.04 & $0.02(-0.0002$ to 0.04$)$ \\
\hline Birthweight (g) : placenta weight (g) & $7.60(6.80,8.41)^{\mathrm{A}}$ & $7.46(6.73,8.29)^{\mathrm{A}}$ & $7.32(6.56,8.12)^{\mathrm{B}}$ & $7.29(6.59,8.26)^{\mathrm{AB}}$ & $<0.0001$ & $-0.03(-0.05$ to -0.006$)$ \\
\hline Birthweight $(\mathrm{g})$ : placental largest diameter $(\mathrm{cm})$ & $164(150,179)^{\mathrm{A}}$ & $167(153,182)^{\mathrm{B}}$ & $169(154,183)^{\mathrm{B}}$ & $167(156,184)^{\mathrm{B}}$ & $<0.0001$ & $0.05(0.03-0.07)$ \\
\hline Birthweight (g) :placental smallest diameter (cm) & $190(172,210)^{\mathrm{A}}$ & $193(177,212)^{\mathrm{B}}$ & $195(180,214)^{\mathrm{B}}$ & $195(178,214)^{\mathrm{B}}$ & $<0.0001$ & $0.04(0.02-0.07)$ \\
\hline Birthweight (g) : placental thickness (mm) & $142(123,163)^{\mathrm{A}}$ & $145(127,167)^{\mathrm{B}}$ & $147(128,170)^{\mathrm{B}}$ & $151(129,174)^{\mathrm{B}}$ & $<0.0001$ & $0.04(0.02-0.06)$ \\
\hline \multicolumn{7}{|l|}{ Cord } \\
\hline Cord number of vessels & & & & & 0.80 & $0.01(0.00-0.02)$ \\
\hline Two & $7(0.58)$ & $51(0.64)$ & $4(0.43)$ & $1(0.35)$ & & \\
\hline Three & $1088(90.6)$ & $7409(92.5)$ & $867(94.0)$ & $260(92.2)$ & & \\
\hline Other/unknown/missing & $106(8.83)$ & $550(6.87)$ & $51(5.53)$ & $21(7.45)$ & & \\
\hline Cord edema & & & & & 0.36 & $0.02(0.00-0.04)$ \\
\hline Not seen & $1059(88.2)$ & $7232(90.3)$ & $837(90.8)$ & $251(89.0)$ & & \\
\hline Present & $16(1.33)$ & $128(1.60)$ & $18(1.95)$ & $8(2.84)$ & & \\
\hline Unknown/missing & $126(10.5)$ & $650(8.11)$ & $67(7.27)$ & $23(8.16)$ & & \\
\hline \multicolumn{7}{|l|}{ Membranes and fetal surface } \\
\hline Opacity of membranes & & & & & 0.06 & $0.02(0.00-0.03)$ \\
\hline Not opaque & $1023(85.2)$ & $6971(87.0)$ & $796(86.3)$ & $242(85.8)$ & & \\
\hline Opaque & $70(5.8)$ & $478(5.97)$ & $73(7.9)$ & $18(6.38)$ & & \\
\hline Partially opaque & 0 & $12(0.15)$ & $4(0.43)$ & & & \\
\hline Unknown/missing & $108(8.99)$ & $549(6.85)$ & $49(5.31)$ & $22(7.80)$ & & \\
\hline Thrombosed fetal vessels & & & & & 0.02 & $0.03(0.003-0.05)$ \\
\hline Not seen & $1096(91.3)$ & 7445 (92.9) & $870(94.4)$ & $259(91.8)$ & & \\
\hline Present & 0 & $23(0.29)$ & $3(0.3)$ & $3(1.06)$ & & \\
\hline Unknown/missing & $105(8.74)$ & $542(6.77)$ & $49(5.31)$ & $20(7.09)$ & & \\
\hline \multicolumn{7}{|l|}{ Cut surface } \\
\hline Infarct size & & & & & 0.45 & $0.02(0.00-0.03)$ \\
\hline Not applicable & $817(68.0)$ & $5440(67.9)$ & $619(67.1)$ & $183(64.9)$ & & \\
\hline
\end{tabular}




\begin{tabular}{|c|c|c|c|c|c|c|}
\hline $\begin{array}{l}\text { All infarcts }<3 \mathrm{cms} \\
>1 \text { infarct measures } \geq 3 \mathrm{~cm} \\
\text { Unknown } / \text { missing }\end{array}$ & $\begin{array}{l}238(19.8) \\
35(2.9) \\
111(9.24)\end{array}$ & $\begin{array}{l}1717(21.4) \\
280(3.50) \\
573(7.15)\end{array}$ & $\begin{array}{l}221(24.0) \\
31(3.36) \\
51(5.53)\end{array}$ & $\begin{array}{l}68(24.1) \\
10(3.55) \\
21(7.45)\end{array}$ & & \\
\hline Cut surface calcification & & & & & 0.18 & $0.02(0.00-0.03)$ \\
\hline None & $461(38.4)$ & $2986(37.3)$ & $340(36.9)$ & $124(44.0)$ & & \\
\hline Maternal surface only & $473(39.4)$ & 3333 (41.6) & $388(42.1)$ & $104(36.8)$ & & \\
\hline Calcification throughout & $158(13.2)$ & $1112(13.9)$ & $144(15.6)$ & $33(11.7)$ & & \\
\hline Unknown/missing & $109(9.08)$ & $579(7.23)$ & $50(5.42)$ & $21(7.45)$ & & \\
\hline
\end{tabular}

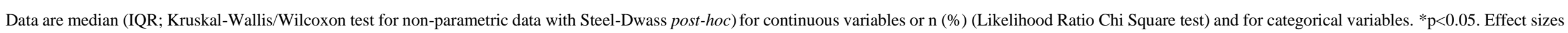
are Wilcoxon test effect size (r) $(95 \% \mathrm{CI})$ for continuous variables, and Cramer's V $(95 \% \mathrm{CI})$ for categorical variables. UW = underweight. NW = normal weight. OW = overweight. OB = obese. 
Supplementary Table 6. Multivariable analyses for associations between maternal prepregnancy BMI (continuous) and macroscopic placental pathologies in term pregnancies, N=10,145.

\begin{tabular}{|c|c|c|c|c|c|c|}
\hline \multirow{2}{*}{ Placental pathology } & \multicolumn{2}{|c|}{ Unadjusted model } & \multicolumn{4}{|c|}{ Adjusted model } \\
\hline & $\beta(95 \% \mathrm{CI})$ & OR $(95 \%$ CI $)$ & p-value & $\beta(95 \% \mathrm{CI})$ & OR $(95 \% \mathrm{CI})$ & p-value \\
\hline \multicolumn{7}{|l|}{ Placenta } \\
\hline Weight (g) & $2.84(2.33-3.36)$ & - & $* * * *$ & $3.71(3.18-4.25)$ & - & $* * * *$ \\
\hline Largest diameter $(\mathrm{cm})$ & $0.04(0.03-0.06)$ & - & $* * * *$ & $0.06(0.04-0.07)$ & - & $* * * *$ \\
\hline Smallest diameter $(\mathrm{cm})$ & $0.04(0.03-0.05)$ & - & $* * * *$ & $0.05(0.03,0.06)$ & - & $* * * *$ \\
\hline Thickness (mm) & $0.007(-0.02$ to 0.03$)$ & - & NS & $0.05(0.02,0.07)$ & - & $* *$ \\
\hline Birthweight (g) : placenta weight (g) & $-0.02(-0.03$ to -0.02$)$ & - & $* * * *$ & $-0.02(-0.03$ to -0.02$)$ & - & $* * * *$ \\
\hline Birthweight $(\mathrm{g})$ : placental largest diameter $(\mathrm{cm})$ & $0.20(0.07-0.33)$ & - & $* *$ & $0.43(0.30-0.57)$ & - & $* * * *$ \\
\hline Birthweight $(\mathrm{g})$ : placental smallest diameter $(\mathrm{cm})$ & $0.28(0.11-0.44)$ & - & $* * *$ & $0.56(0.39-0.73)$ & - & $* * * *$ \\
\hline Birthweight $(\mathrm{g})$ : placental thickness (mm) & $0.55(0.35-0.76)$ & - & $* * * *$ & $0.56(0.34-0.77)$ & - & $* * * *$ \\
\hline \multicolumn{7}{|l|}{ Cord } \\
\hline $\begin{array}{l}\text { Cord number of vessels } \\
\text { Two } \\
\text { Three }\end{array}$ & $-0.02(-0.10$ to 0.06$)$ & $0.98(0.90-1.06)$ & NS & $-0.03(-0.12$ to 0.06$)$ & $0.97(0.89-1.06)$ & NS \\
\hline $\begin{array}{l}\text { Cord edema } \\
\text { Not seen } \\
\text { Present }\end{array}$ & $0.03(-0.01$ to 0.07$)$ & $1.03(0.99-1.07)$ & NS & $0.02(-0.03$ to 0.07$)$ & $1.02(0.97-1.07)$ & NS \\
\hline \multicolumn{7}{|l|}{ Membranes and fetal surface } \\
\hline $\begin{array}{l}\text { Opacity of membranes } \\
\text { Not opaque } \\
\text { Any degree of opacity }\end{array}$ & $0.02(-0.002$ to 0.04$)$ & $1.02(1.00-1.05)$ & NS & $0.02(-0.008$ to 0.04$)$ & $1.02(0.99-1.04)$ & NS \\
\hline $\begin{array}{l}\text { Thrombosed fetal vessels } \\
\text { Not seen } \\
\text { Present }\end{array}$ & $0.06(-0.02$ to 0.15$)$ & $1.07(0.98-1.16)$ & NS & $0.05(-0.06$ to 0.16$)$ & $1.05(0.94-1.18)$ & NS \\
\hline \multicolumn{7}{|l|}{ Cut surface } \\
\hline $\begin{array}{l}\text { Infarcts } \\
\text { Not applicable } \\
\text { Any infarcts seen }\end{array}$ & $0.02(0.006-0.03)$ & $1.02(1.01-1.03)$ & $* *$ & $0.03(0.02-0.04)$ & $1.03(1.02-1.05)$ & $* * * *$ \\
\hline $\begin{array}{l}\text { Cut surface calcification } \\
\text { None } \\
\text { Any calcification seen }\end{array}$ & $-0.004(-0.02$ to 0.009$)$ & $1.00(0.98-1.01)$ & NS & $0.002(-0.01$ to 0.02$)$ & $1.00(0.99-1.02)$ & NS \\
\hline
\end{tabular}

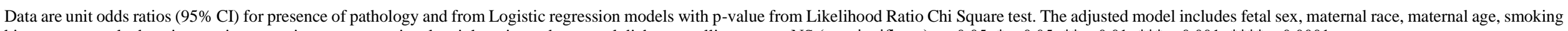
history, maternal education, socioeconomic status, gestational weight gain, and maternal diabetes mellitus status. NS (not significant): $\mathrm{p}>0.05 ;{ }^{*} \mathrm{p} \leq 0.05 ; * * \mathrm{p} \leq 0.01 ; * * * \mathrm{p} \leq 0.001 ; * * * * \mathrm{p} \leq 0.0001$. 
Supplementary Table 7. Associations between maternal prepregnancy BMI and macroscopic placental pathologies in preterm pregnancies, N=1739.

\begin{tabular}{|c|c|c|c|c|c|c|}
\hline \multirow[t]{2}{*}{ Placental pathology } & \multicolumn{4}{|c|}{ Prepregnancy BMI } & \multirow[b]{2}{*}{ p-value } & \multirow[b]{2}{*}{ Effect size } \\
\hline & $\begin{array}{l}\text { UW } \\
(\mathrm{n}=259)\end{array}$ & $\begin{array}{l}\text { NW } \\
(n=1286)\end{array}$ & $\begin{array}{l}\text { OW } \\
(n=156)\end{array}$ & $\begin{array}{l}\text { OB } \\
(\mathrm{n}=38)\end{array}$ & & \\
\hline \multicolumn{7}{|l|}{ Placenta } \\
\hline $\begin{array}{l}\text { Placenta shape } \\
\text { Non-partite } \\
\text { Bipartite } \\
\text { Tripartite } \\
\text { Succenturiate } \\
\text { Membranous placenta } \\
\text { Crescent shaped } \\
\text { Irregular shape } \\
\text { Unknown/missing }\end{array}$ & $\begin{array}{l}219(84.6) \\
0 \\
0 \\
5(1.9) \\
0 \\
0 \\
0 \\
35(13.5)\end{array}$ & $\begin{array}{l}1135(88.4) \\
5(0.39) \\
0 \\
16(1.24) \\
0 \\
0 \\
7(0.54) \\
123(9.56)\end{array}$ & $\begin{array}{l}142(91.0) \\
0 \\
0 \\
1(0.64) \\
0 \\
0 \\
0 \\
13(8.33)\end{array}$ & $\begin{array}{l}35(92.1) \\
0 \\
0 \\
1(2.63) \\
0 \\
0 \\
0 \\
2(5.26)\end{array}$ & 0.43 & $0.04(0.00-0.04)$ \\
\hline Weight $(\mathrm{g})$ & $360(310,425)^{\mathrm{A}}$ & $380(320,440)^{\mathrm{AB}}$ & $405(330,470)^{\mathrm{B}}$ & $440(360,501)^{\mathrm{B}}$ & 0.0008 & $0.04(-0.006$ to 0.09$)$ \\
\hline Largest diameter $(\mathrm{cm})$ & $18(16,19)^{\mathrm{A}}$ & $18(17,19)^{\mathrm{A}}$ & $18(17,20)^{\mathrm{A}}$ & $19(16,21)^{\mathrm{A}}$ & 0.05 & $0.05(-0.004$ to 0.11$)$ \\
\hline Smallest diameter $(\mathrm{cm})$ & $15(14,17)^{\mathrm{A}}$ & $16(14,17)^{\mathrm{AB}}$ & $16(14,17)^{\mathrm{AB}}$ & $17(15,18)^{\mathrm{B}}$ & 0.005 & $0.06(0.009-0.12)$ \\
\hline Thickness (mm) & $20(18,25)$ & $20(18,23)$ & $20(18,25)$ & $20(19,25)$ & 0.77 & $-0.0006(-0.05$ to 0.05$)$ \\
\hline Birthweight $(\mathrm{g})$ : placenta weight $(\mathrm{g})$ & $7.34(6.49,8.31)$ & $7.22(6.45,8.11)$ & $7.21(6.41,7.91)$ & $6.99(6.21,8.26)$ & 0.49 & $-0.03(-0.07$ to 0.02$)$ \\
\hline Birthweight $(\mathrm{g})$ : placental largest diameter $(\mathrm{cm})$ & $151(138,167)$ & $154(136,170)$ & $158(140,173)$ & $163(146,177)$ & 0.23 & $0.02(-0.03$ to 0.06$)$ \\
\hline Birthweight $(\mathrm{g})$ : placental smallest diameter $(\mathrm{cm})$ & $174(161,195)$ & $178(159,198)$ & $180(162,204)$ & $183(171,204)$ & 0.52 & $0.02(-0.04$ to 0.07$)$ \\
\hline Birthweight $(\mathrm{g})$ : placental thickness $(\mathrm{mm})$ & $134(115,156)$ & $135(117,159)$ & $140(119,159)$ & $147(119,170)$ & 0.23 & $0.03(-0.02$ to 0.08$)$ \\
\hline \multicolumn{7}{|l|}{ Cord } \\
\hline $\begin{array}{l}\text { Cord number of vessels } \\
\text { Two } \\
\text { Three } \\
\text { Other/unknown/missing }\end{array}$ & $\begin{array}{l}1(0.39) \\
223(86.1) \\
35(13.5)\end{array}$ & $\begin{array}{l}10(0.78) \\
1154(89.7) \\
122(9.49)\end{array}$ & $\begin{array}{l}1(0.64) \\
143(91.7) \\
12(7.69)\end{array}$ & $\begin{array}{l}0 \\
37(97.4) \\
1(2.63)\end{array}$ & 0.79 & $0.02(0.00-0.05)$ \\
\hline $\begin{array}{l}\text { Cord edema } \\
\quad \text { Not seen } \\
\text { Present } \\
\text { Unknown/missing } \\
\end{array}$ & $\begin{array}{l}218(84.2) \\
5(1.93) \\
36(13.9)\end{array}$ & $\begin{array}{l}1099(85.5) \\
50(3.90) \\
137(10.7)\end{array}$ & $\begin{array}{l}131(84.0) \\
10(6.41) \\
15(9.62)\end{array}$ & $\begin{array}{l}33(86.8) \\
3(7.89) \\
2(5.26) \\
\end{array}$ & 0.11 & $0.06(0.00-0.11)$ \\
\hline \multicolumn{7}{|l|}{ Membranes and fetal surface } \\
\hline $\begin{array}{l}\text { Opacity of membranes } \\
\text { Not opaque } \\
\text { Opaque } \\
\text { Partially opaque } \\
\text { Unknown/missing }\end{array}$ & $\begin{array}{l}208(80.3) \\
15(5.79) \\
0 \\
36(13.9)\end{array}$ & $\begin{array}{l}1078(83.8) \\
86(6.69) \\
0 \\
122(9.49) \\
\end{array}$ & $\begin{array}{l}129(82.7) \\
14(8.97) \\
1(0.64) \\
12(7.69)\end{array}$ & $\begin{array}{l}32(84.2) \\
5(13.2) \\
0 \\
1(2.63)\end{array}$ & 0.28 & $0.06(0.00-0.09)$ \\
\hline $\begin{array}{l}\text { Thrombosed fetal vessels } \\
\text { Not seen } \\
\text { Present } \\
\text { Unknown/missing }\end{array}$ & $\begin{array}{l}221(85.3) \\
3(1.16) \\
35(13.5)\end{array}$ & $\begin{array}{l}1160(90.2) \\
7(0.54) \\
119(9.25)\end{array}$ & $\begin{array}{l}141(90.4) \\
3(1.92) \\
12(7.69)\end{array}$ & $\begin{array}{l}37(97.4) \\
0 \\
1(2.63)\end{array}$ & 0.26 & $0.05(0.00-0.09)$ \\
\hline \multicolumn{7}{|l|}{ Cut Surface } \\
\hline $\begin{array}{l}\text { Infarct size } \\
\text { Not applicable } \\
\text { All infarcts }<3 \mathrm{cms} \\
\text { At least one infarct measures } \geq 3 \mathrm{cms}\end{array}$ & $\begin{array}{l}178(68.7) \\
34(13.1) \\
9(3.47)\end{array}$ & $\begin{array}{l}936(72.8) \\
191(14.9) \\
39(3.03)\end{array}$ & $\begin{array}{l}111(71.2) \\
28(17.9) \\
5(3.21)\end{array}$ & $\begin{array}{l}28(73.7) \\
7(18.4) \\
1(2.63)\end{array}$ & 0.95 & $0.02(0.00-0.02)$ \\
\hline
\end{tabular}




\begin{tabular}{|c|c|c|c|c|c|c|}
\hline Unknown/missing & $38(14.7)$ & $120(9.33)$ & $12(7.69)$ & $2(5.26)$ & & \\
\hline Cut surface calcification & & & & & 0.74 & $0.03(0.00-0.05)$ \\
\hline None & $119(45.9)$ & $638(49.6)$ & $85(54.5)$ & $16(42.1)$ & & \\
\hline Maternal surface only & $78(30.1)$ & $396(30.8)$ & $42(26.9)$ & $14(36.8)$ & & \\
\hline Calcification throughout & $25(9.65)$ & $129(10.0)$ & $17(10.9)$ & $6(15.8)$ & & \\
\hline Unknown/missing & $37(14.3)$ & $123(9.56)$ & $12(7.69)$ & $2(5.26)$ & & \\
\hline
\end{tabular}

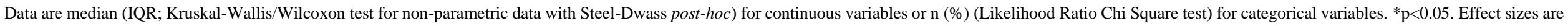
Wilcoxon test effect size (r) $(95 \% \mathrm{CI})$ for continuous variables, or Cramer's V $(95 \% \mathrm{CI})$ for categorical variables. $\mathrm{UW}=$ underweight. $\mathrm{NW}=$ normal weight. OW $=$ overweight. OB $=$ obese. 
Supplementary Table 8. Multivariable analyses for associations between maternal prepregnancy BMI (continuous) and macroscopic placental pathologies in preterm pregnancies, N=1739.

\begin{tabular}{|c|c|c|c|c|c|c|}
\hline \multirow[t]{2}{*}{ Placental pathology } & \multicolumn{2}{|c|}{ Unadjusted model } & \multicolumn{4}{|c|}{ Adjusted model } \\
\hline & $\beta(95 \% \mathrm{CI})$ & OR $(95 \% \mathrm{CI})$ & p-value & $\beta(95 \% \mathrm{CI})$ & OR $(95 \% \mathrm{CI})$ & p-value \\
\hline \multicolumn{7}{|l|}{ Placenta } \\
\hline Weight (g) & $3.26(1.80-4.73)$ & - & $* * * *$ & $3.25(1.72-4.77)$ & - & $* * * *$ \\
\hline Largest diameter $(\mathrm{cm})$ & $0.05(0.01-0.08)$ & - & $* *$ & $0.04(0.01-0.08)$ & - & $* *$ \\
\hline Smallest diameter $(\mathrm{cm})$ & $0.06(0.03-0.09)$ & - & $* * *$ & $0.06(0.03-0.09)$ & - & $* * *$ \\
\hline Thickness (mm) & $0.05(-0.02$ to 0.11$)$ & - & NS & $0.06(-0.007$ to 0.13$)$ & - & NS \\
\hline Birthweight (g) : placenta weight (g) & $-0.02(-0.04$ to 0.006$)$ & - & NS & $-0.01(-0.04$ to 0.02$)$ & - & NS \\
\hline Birthweight $(\mathrm{g})$ : placental largest diameter $(\mathrm{cm})$ & $0.62(0.22-1.02)$ & - & $* *$ & $0.87(0.45-1.29)$ & - & $* * * *$ \\
\hline Birthweight $(\mathrm{g})$ : placental smallest diameter $(\mathrm{cm})$ & $0.37(-0.11$ to 0.85$)$ & - & NS & $0.67(0.15-1.18)$ & - & $* *$ \\
\hline Birthweight $(\mathrm{g})$ : placental thickness $(\mathrm{mm})$ & $0.53(-0.04$ to 1.10$)$ & - & NS & $0.49(-0.12$ to 1.10$)$ & - & NS \\
\hline \multicolumn{7}{|l|}{ Cord } \\
\hline $\begin{array}{l}\text { Cord number of vessels } \\
\text { Two } \\
\text { Three }\end{array}$ & $0.03(-0.14$ to 0.19$)$ & $1.03(0.87-1.21)$ & NS & $0.04(-0.13$ to 0.22$)$ & $1.04(0.88-1.24)$ & NS \\
\hline $\begin{array}{l}\text { Cord edema } \\
\text { Not seen } \\
\text { Present }\end{array}$ & $0.07(0.003-0.13)$ & $1.07(1.00-1.14)$ & NS & $0.04(-0.03$ to 0.11$)$ & $1.04(0.97-1.12)$ & NS \\
\hline \multicolumn{7}{|l|}{ Membranes and fetal surface } \\
\hline $\begin{array}{l}\text { Opacity of membranes } \\
\text { Not opaque } \\
\text { Any degree of opacity }\end{array}$ & $0.05(0.005-0.10)$ & $1.06(1.01-1.11)$ & $*$ & $0.05(-0.007$ to 0.11$)$ & $1.05(0.99-1.11)$ & NS \\
\hline $\begin{array}{l}\text { Thrombosed fetal vessels } \\
\text { Not seen } \\
\text { Present }\end{array}$ & $0.04(-0.12$ to 0.19$)$ & 1.04 (0.89 to 1.20$)$ & NS & $0.02(-0.14$ to 0.18$)$ & $1.02(0.87-1.19)$ & NS \\
\hline \multicolumn{7}{|l|}{ Cut Surface } \\
\hline $\begin{array}{l}\text { Infarcts } \\
\text { Not applicable } \\
\text { Any infarcts seen }\end{array}$ & $0.03(-0.005$ to 0.07$)$ & $1.03(1.00-1.07)$ & NS & $0.03(-0.006$ to 0.08$)$ & $1.04(0.99-1.08)$ & NS \\
\hline $\begin{array}{l}\text { Cut surface calcification } \\
\text { None } \\
\text { Any calcification seen }\end{array}$ & $-0.002(-0.03$ to 0.03$)$ & $1.0 \quad(0.97-1.03)$ & NS & $-0.009(-0.04$ to 0.02$)$ & $0.99(0.96-1.03)$ & NS \\
\hline
\end{tabular}

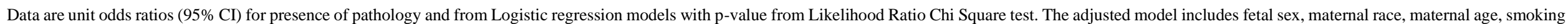
history, maternal education, socioeconomic status, gestational weight gain, and maternal diabetes mellitus status. NS (not significant): $\mathrm{p}>0.05 ;{ }^{*} \mathrm{p} \leq 0.05 ; * * \mathrm{p} \leq 0.01 ; * * * \mathrm{p} \leq 0.001 ; * * * * \mathrm{p} \leq 0.0001$. 
medRxiv preprint doi: https://doi.org/10.1101/2021.06.01.21258127; this version posted June 4, 2021. The copyright holder for this preprint (which was not certified by peer review) is the author/funder, who has granted medRxiv a license to display the preprint in perpetuity.

All rights reserved. No reuse allowed without permission.

\section{Supplementary figures}

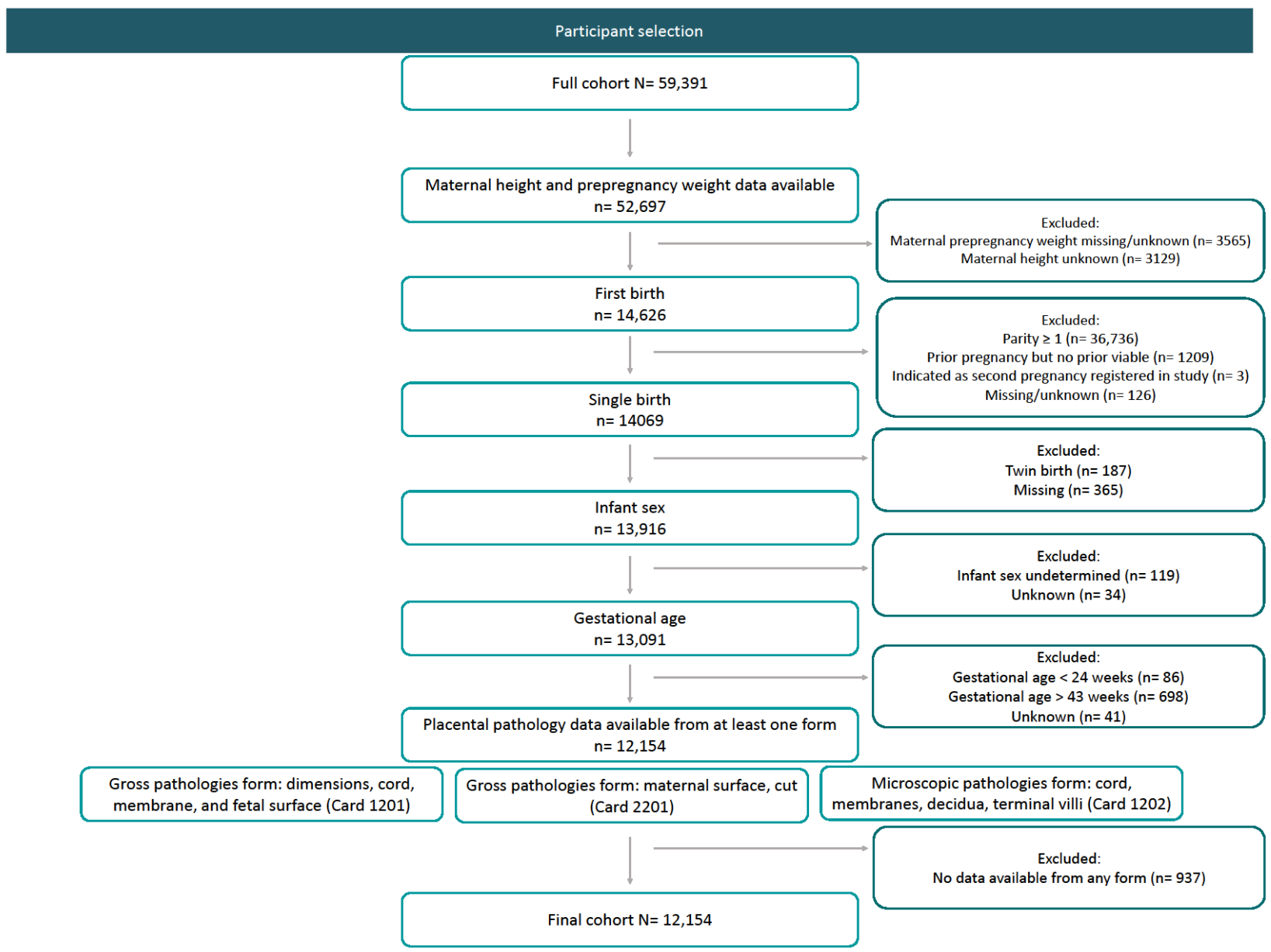

Cohort stratification

Term pregnancies $n=10,145$



Preterm pregnancies $n=1739$

OW
$n=1286$$\quad \begin{gathered}\text { OB } \\ n=38\end{gathered}$

Supplementary Figure 1. Participant flow selection and placental pathologies of interest. 
medRxiv preprint doi: https://doi.org/10.1101/2021.06.01.21258127; this version posted June 4, 2021. The copyright holder for this preprint (which was not certified by peer review) is the author/funder, who has granted medRxiv a license to display the preprint in perpetuity.

All rights reserved. No reuse allowed without permission.

A

Preterm

Term

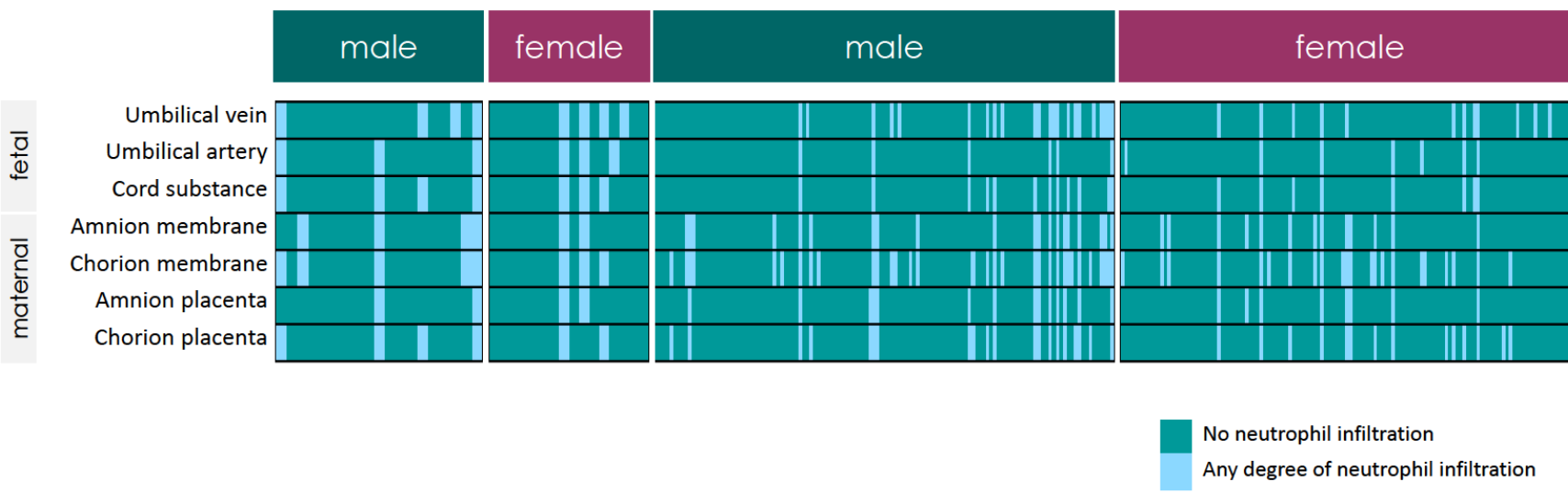

B

\begin{tabular}{|c|c|c|c|c|c|c|c|c|}
\hline \multirow[b]{2}{*}{ Neutrophil infiltration } & \multicolumn{2}{|c|}{ Preterm } & \multicolumn{6}{|c|}{ Term } \\
\hline & $\begin{array}{l}\text { Male } \\
(n=22)\end{array}$ & $\begin{array}{l}\text { Female } \\
(n=16)\end{array}$ & OR $(95 \% \mathrm{Cl})$ & $\mathrm{p}$-value & $\begin{array}{l}\text { Male } \\
(n=135)\end{array}$ & $\begin{array}{l}\text { Female } \\
(n=147)\end{array}$ & OR $(95 \% \mathrm{Cl})$ & $\mathrm{p}$-value \\
\hline Umbilical vein & & & $1.00(0.22,4.56)$ & NS & & & $2.08(1.03,4.22)$ & 0.04 \\
\hline Not seen & $15(68.2)$ & $12(75.0)$ & & & $107(79.3)$ & $130(88.4)$ & & \\
\hline Any degree & $5(22.7)$ & $4(25.0)$ & & & $24(17.8)$ & $14(9.52)$ & & \\
\hline Unknown/missing & $2(9.09)$ & 0 & & & $4(2.96)$ & $3(2.04)$ & & \\
\hline Umbilical artery & & & $0.76(0.13,4.43)$ & NS & & & $0.96(0.34,2.72)$ & NS \\
\hline Not seen & $17(77.3)$ & $13(81.3)$ & & & 124 (91.9) & $136(92.5)$ & & \\
\hline Any degree & $3(13.6)$ & $3(18.8)$ & & & $7(5.19)$ & $8(5.44)$ & & \\
\hline Unknown/missing & $2(9.09)$ & 0 & & & $4(2.96)$ & $3(2.04)$ & & \\
\hline Umbilical cord substance & & & $1.08(0.20,5.73)$ & NS & & & $2.03(0.82,5.02)$ & NS \\
\hline Not seen & $16(72.7)$ & $13(81.3)$ & & & $117(86.7)$ & $136(92.5)$ & & \\
\hline Any degree & $4(18.2)$ & $3(18.8)$ & & & $14(10.4)$ & $8(5.44)$ & & \\
\hline Unknown/missing & $2(9.09)$ & 0 & & & $4(2.96)$ & $3(2.04)$ & & \\
\hline Amnion membrane & & & $2.33(0.39,14.0)$ & NS & & & $1.53(0.75,3.12)$ & NS \\
\hline Not seen & $15(68.2)$ & $14(87.5)$ & & & $107(79.3)$ & $117(79.6)$ & & \\
\hline Any degree & $5(22.7)$ & $2(12.5)$ & & & $21(15.6)$ & $15(10.2)$ & & \\
\hline Unknown/missing & $2(9.09)$ & 0 & & & $7(5.19)$ & $15(10.2)$ & & \\
\hline Chorion membrane & & & $1.86(0.38,9.00)$ & NS & & & $1.88(1.05,3.36)$ & 0.03 \\
\hline Not seen & $14(63.6)$ & $13(81.3)$ & & & $96(71.1)$ & $120(81.6)$ & & \\
\hline Any degree & $6(27.3)$ & $3(18.8)$ & & & $36(26.7)$ & $24(16.3)$ & & \\
\hline Unknown/missing & $2(9.09)$ & 0 & & & $3(2.22)$ & $3(2.04)$ & & \\
\hline Amnion placenta & & & $0.82(0.10,6.62)$ & NS & & & $1.92(0.78,4.75)$ & NS \\
\hline Not seen & $17(77.3)$ & $14(87.5)$ & & & $113(83.7)$ & $124(84.4)$ & & \\
\hline Any degree & $2(9.09)$ & $2(12.5)$ & & & $14(10.4)$ & $8(5.44)$ & & \\
\hline Unknown/missing & $3(13.6)$ & 0 & & & $8(5.93)$ & $15(10.2)$ & & \\
\hline Chorion placenta & & & $2.33(0.39,14.0)$ & NS & & & $1.72(0.86,3.54)$ & NS \\
\hline Not seen & $15(68.2)$ & $14(87.5)$ & & & $110(81.5)$ & $129(87.8)$ & & \\
\hline Any degree & $5(22.7)$ & $2(12.5)$ & & & $22(16.3)$ & $15(10.2)$ & & \\
\hline Unknown/missing & 2 (9.09) & 0 & & & $3(2.22)$ & $3(2.04)$ & & \\
\hline
\end{tabular}

Supplementary Figure 2. Placental neutrophil infiltration in pregnancies with maternal obesity by fetal sex, $n=320$. Prescence or absence of neutrophil infiltration for female and male placentae among pregnancies with maternal obesity by preterm (male: $n=22$, female: $n=16$ ) and term (male: $n=135$, female: $n=147$ ) birth. 